\title{
New Mn II energy levels from the STIS-HST spectrum of the HgMn star HD 175640^
}

\author{
F. Castelli ${ }^{1}$, R. L. Kurucz ${ }^{2}$, and C. R. Cowley ${ }^{3}$ \\ ${ }^{1}$ Istituto Nazionale di Astrofisica, Osservatorio Astronomico di Trieste, via Tiepolo 11, 34143 Trieste, Italy \\ e-mail: castelli@oats.inaf.it \\ 2 Harvard-Smithsonian Center for Astrophysics, 60 Garden Street, Cambridge, MA 02138, USA \\ e-mail: rkurucz@cfa.harvard.edu \\ 3 Department of Astronomy, University of Michigan, Ann Arbor, MI 48109-1042, USA \\ e-mail: cowley@umich.edu
}

Received 15 February 2015 / Accepted 6 April 2015

\begin{abstract}
Aims. The NIST database lists several Mn II lines that were observed in the laboratory but not classified. They cannot be used in spectrum synthesis because their atomic line data are unknown. These lines are concentrated in the 2380-2700 ̊ interval. We aimed to assign energy levels and $\log g f$ values to these lines.

Methods. Semi-empirical line data for Mn II computed by Kurucz were used to synthesize the ultraviolet spectrum of the slowrotating, HgMn star HD 175640. The spectrum was compared with the high-resolution spectrum observed with the HST-STIS equipment. A UVES spectrum covering the 3050-10000 A region was also examined.

Results. We determined a total of 73 new energy levels, 58 from the STIS spectrum of HD 175640 and another 15 from the UVES spectrum. The new energy levels give rise to numerous new computed lines. We have identified more than $50 \%$ of the unclassified lines listed in the NIST database and have changed the assignment of another 24 lines. An abundance analysis of the star HD 175640, based on the comparison of observed and computed ultraviolet spectra in the 1250-3040 A interval, is the by-product of this study on Mn II.
\end{abstract}

Key words. line: identification - atomic data - stars: atmospheres - stars: chemically peculiar - stars: individual: HD 175640

\section{Introduction}

The availability of a reliable and complete set of atomic data is an essential requirement for research in stellar physics, in particular for computing stellar atmospheres and stellar spectra to be compared with observations at various resolutions.

The high-resolution, high signal-to-noise stellar spectra observed by the modern telescopes and spectrographs have proved to be a useful tool for studies aimed at extending the knowledge of atomic and molecular data. An example is the analysis of the UVES spectrum of the peculiar star HR 6000 which has permitted us to fix about $120 \mathrm{Fe}$ II energy levels not observed before in the laboratory, but predicted with approximate energy values by the theory (Castelli et al. 2008, 2009; Castelli \& Kurucz 2010). Another example of the use of stellar spectra to derive atomic data is the paper by Peterson \& Kurucz (2015) who obtained 66 new energy levels for Fe I from the analysis of HST-STIS spectra of a group of 13 metal-poor stars. In the above works, for each new determined energy level, several new transitions with wavelengths and oscillator strengths are associated, so that the line lists used for computing spectra are considerably augmented.

In this paper we extend to Mn II the above described studies. History has shown that the refinement and extension of our atomic database has lead to important astronomical discoveries.

\footnotetext{
* Tables A.1 and A.2 are only available at the CDS via anonymous ftp to cdsarc.u-strasbg. fr $(130.79 .128 .5)$ or via http://cdsarc.u-strasbg.fr/viz-bin/qcat?]/A+A/580/A10
}

In the case of the Mn II spectrum, the subject of the present paper, the extended analysis by Iglesias \& Velasco (1964) enabled many new line identifications in the class of stars now known as mercury-manganese $(\mathrm{HgMn})$ stars, occupying the spectral region $\sim$ A0/B9-B6 $(10500-16000 \mathrm{~K})$. This in turn enabled Bidelman (1962) and Dworetsky (1969) to show that these stars were enormously enriched in mercury and platinum. In the present study, we have been able to obtain abundances for ten elements that were unavailable from the ground spectra $(\mathrm{B}, \mathrm{N}$, $\mathrm{Al}, \mathrm{Cl}, \mathrm{V}, \mathrm{Zn}, \mathrm{Ge}, \mathrm{As}, \mathrm{Ag}$, and $\mathrm{Cd}$ ). The ability to distinguish their spectral lines from those of Mn II was essential.

To study the Mn II spectrum, we adopted the same procedure described by Castelli \& Kurucz (2010). We analysed HST-STIS spectra from 1250-3045 $\AA$ and a UVES spectrum from 3050 to $10000 \AA$ of the HgMn star HD 175640. It is a slowly rotating star with an abundance in manganese on the order of 2.5 dex over the solar value. The UVES spectrum, previously analyzed to derive stellar parameters and abundances by Castelli \& Hubrig (2004), was further examined for this paper to derive new Mn II energy levels.

To fix new energy levels we adopted the semi-empirical Mn II line data computed by Kurucz. We determined a total of 73 new energy levels, 58 from the HST-STIS spectrum and 15 from the UVES spectrum. Updated line lists for most elements were adopted in order to synthesize the spectra. The most recent work on Mn II is that of Kramida \& Sansonetti (2013) who critically analyzed all the atomic data available in the literature for this ion. They revised the Sugar \& Corliss (1985) energy levels and 
provided a list of Mn II spectral lines and transition probabilities taken mostly from experimental sources. The Mn II atomic data of the NIST database (Kramida et al. 2014) are the result of the Kramida \& Sansonetti (2013) work. In the database there are numerous unclassified lines of Mn II. They are concentrated in the 2380-2700 $\AA$ interval which was observed for HD 175640 at about 120000 resolution. The new Mn II energy levels have permitted us to identify more than $50 \%$ of the lines unclassified in the NIST database.

\section{Observations and data reduction}

HD 175640 (HR 7143) is one of the targets included in the "Hot Stars" program (GO-13346), which is part of the "Advanced Spectral Library (ASTRAL)" Project (Ayres 2014). The star was observed in Cycle 21 of the Space Telescope Imaging Spectrograph (STIS). The spectrum covers the range 1150-3045 A. The nominal resolving power $\mathrm{R}$ ranges from about 30000 to 120000 ; signal-to-noise ratio is larger than 100 . We used the final spectrum that resulted from the calibration and the merging of the individual spectra observed in the different wavelength intervals, as performed by the ASTRAL Science Team (Carpenter et al. 2015).

For this work we analyzed the whole region from 1250 to $3045 \AA$. We used the IRAF tool "continuum" to normalize the observed spectrum to the continuum level. When we compared observed and computed spectra we tentatively fixed different resolving powers for the different spectral intervals roughly corresponding to the different resolutions of the different observed regions (Ayres 2010). In particular, we adopted $\mathrm{R}$ equal to $50000,40000,25000,30000$, and 120000 for the ranges

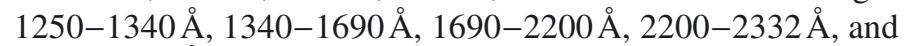
2332-3045 A, respectively. The observed spectrum was shifted to the Ritz wavelengths, as derived from the energy levels, using mostly Fe II lines as reference lines, owing to their accurate wavelengths derived from the Nave \& Johansson (2013) energy levels. The shift to superimpose the observed spectrum to the synthetic spectrum is $+25 \mathrm{~km} \mathrm{~s}^{-1}$ in the $1250-2000 \AA$ region. After conversion of the wavelength scale of the 2000-3040 $\AA$ interval from vacuum to air, the shift is $+24.5 \mathrm{~km} \mathrm{~s}^{-1}$. We estimate an uncertainty in the velocity shift on the order of $0.5 \mathrm{~km} \mathrm{~s}^{-1}$, corresponding to an uncertainty in the wavelength scale of $0.2 \mathrm{~m} \AA, 0.35 \mathrm{~m} \AA$, and $0.5 \mathrm{~m} \AA$ at $1250 \AA, 2000 \AA$, and $3000 \AA$, respectively.

\section{The star HD 175640}

The stellar parameters and the abundances of HD 175640 were determined by Castelli \& Hubrig (2004) in a previous analysis of the optical spectrum of the star. An ATLAS12 (Kurucz 2005) model with parameters $T_{\text {eff }}=12000 \mathrm{~K}, \log g=3.95$, and microturbulent velocity $\xi=0 \mathrm{~km} \mathrm{~s}^{-1}$ was computed for the individual stellar abundances. They were obtained from the equivalent widths of selected lines and are listed in the third column of Table 1. The SYNTHE code (Kurucz 2005) was used to compute a synthetic spectrum for the 3050-10000 ̊ region which was compared with the observed spectrum. The ATLAS12 model and the given set of abundances were adopted. A rotational velocity of $v \sin i=2.5 \mathrm{~km} \mathrm{~s}^{-1}$ was derived from the comparison of the observed and computed spectra. The adopted zero microturbulent velocity was based both on the Adelman (1994) conclusions that most $\mathrm{HgMn}$ stars have little or no microturbulent velocity and on the consistent abundances we obtained from the
Table 1. Abundances $\log \left(N_{\text {elem }} / N_{\text {tot }}\right)$ for HD 175640 [12000 K, 3.95] adopted to compute the ultraviolet synthetic spectrum.

\begin{tabular}{|c|c|c|c|}
\hline \multirow[t]{2}{*}{ Elem. } & \multicolumn{2}{|c|}{ HD 175640} & \multirow[t]{2}{*}{ Sun } \\
\hline & UV & Visible & \\
\hline He I & & -1.73 & -1.11 \\
\hline Be II & & -10.64 & -10.99 \\
\hline B II & -8.75 & & -9.34 \\
\hline CI & $<-4.00$ & $-4.11 \pm 0.23$ & -3.61 \\
\hline $\mathrm{C}_{\text {II }}$ & -4.00 & $-4.05 \pm 0.16$ & -3.61 \\
\hline N I & -5.95 & $\leq-5.78$ & -4.21 \\
\hline OI & -3.28 & $-3.18 \pm 0.11$ & -3.35 \\
\hline $\mathrm{Ne} I$ & & -4.35 & -4.11 \\
\hline $\mathrm{NaI}$ & & -5.47 & -5.80 \\
\hline Mg I & -4.69 & $-4.64 \pm 0.06$ & -4.44 \\
\hline Mg II & -4.69 & $-4.71 \pm 0.07$ & -4.44 \\
\hline $\mathrm{Al} \mathrm{I}$ & & $<-7.50$ & -5.59 \\
\hline Al II & -7.00 & & -5.59 \\
\hline $\mathrm{Al}$ III & -7.00 & & -5.59 \\
\hline Si I & -4.80 & & -4.53 \\
\hline Si II & -4.80 & $-4.72 \pm 0.08$ & -4.53 \\
\hline Si III & -4.80 & $-4.58 \pm 0.04$ & -4.53 \\
\hline Si IV & -4.80 & & -4.53 \\
\hline PI & $<-6.28$ & & -6.63 \\
\hline P II & -6.28 & $-6.28 \pm 0.08$ & -6.63 \\
\hline P III & -6.28 & & -6.63 \\
\hline S I & -5.30 & & -4.92 \\
\hline S II & -5.30 & $-5.12 \pm 0.03$ & -4.92 \\
\hline $\mathrm{Cl}$ I & -7.50 & & -6.54 \\
\hline $\mathrm{CaI}$ & & -5.26 & -5.70 \\
\hline Ca II & -5.54 & $-5.67 \pm 0.25$ & -5.70 \\
\hline Sc II & -9.08 & $-9.08 \pm 0.15$ & -8.89 \\
\hline Sc III & -9.08 & & -8.89 \\
\hline Ti II & -5.67 & $-5.67 \pm 0.11$ & -7.09 \\
\hline Ti III & -5.67 & & -7.09 \\
\hline V II & -9.94 & $\leq-9.04$ & -8.11 \\
\hline Cr I & & $-5.22 \pm 0.09$ & -6.40 \\
\hline Cr II & -5.36 & $-5.41 \pm 0.07$ & -6.40 \\
\hline Cr III & -5.36 & & -6.40 \\
\hline Mn I & -4.20 & $-4.20 \pm 0.08$ & -6.61 \\
\hline Mn II & -4.20 & $-4.25 \pm 0.04$ & -6.61 \\
\hline Mn III & $-4.20:$ & & -6.61 \\
\hline Fe I & -4.83 & $-4.78 \pm 0.08$ & -4.54 \\
\hline $\mathrm{Fe}$ II & -4.83 & $-4.84 \pm 0.13$ & -4.54 \\
\hline Fe III & -4.83 & & -4.54 \\
\hline Co II & -9.00 & $-8.08:$ & -7.05 \\
\hline Ni II & -6.09 & $-6.09 \pm 0.16$ & -5.82 \\
\hline Ni III & -6.09 & & -5.82 \\
\hline $\mathrm{Cu} \mathrm{I}$ & & -6.52 & -7.85 \\
\hline $\mathrm{Cu}$ II & -6.50 & & -7.85 \\
\hline Zn II & -8.70 & & -7.48 \\
\hline Ga I & -5.43 & & -9.00 \\
\hline Ga II & -5.43 & $-5.43 \pm 0.04$ & -9.00 \\
\hline Ga III & $<-5.43 ?$ & & -9.00 \\
\hline Ge II & -10.1 & & -8.39 \\
\hline As II & -7.50 & & -9.74 \\
\hline $\mathrm{Br}$ II & & $-7.12 \pm 0.04$ & -9.50 \\
\hline Sr II & & -8.41 & -9.17 \\
\hline Y II & -6.66 & $-6.66 \pm 0.20$ & -9.83 \\
\hline Y III & $>-6.66$ & & -9.83 \\
\hline
\end{tabular}

Notes. Solar abundances are from Asplund et al. (2009).

equivalent widths of 55 weak and strong Fe II lines measured in the optical region (Castelli \& Hubrig 2004).

In this paper we compared the observed HST-STIS spectrum with a synthetic spectrum computed for the $1250-3045 \AA$ 
Table 1. continued.

\begin{tabular}{lccc}
\hline \hline Elem & \multicolumn{2}{c}{ HD175640 } & \multicolumn{1}{c}{ Sun } \\
& UV & Visible & \\
\hline Zr II & & $-8.67 \pm 0.17$ & -9.46 \\
Zr III & -8.50 & & -9.46 \\
Rh II & -8.50 & -8.50 & -11.13 \\
Pd I & & $-6.41 \pm 0.30$ & -10.47 \\
Pd II & -6.30 & & -10.47 \\
Pd III & -6.30 & & -10.47 \\
Ag II & -8.50 & & -11.10 \\
Cd II & -9.53 & & -10.33 \\
Xe II & & $-5.96 \pm 0.20$ & -9.80 \\
Ba II & & -9.27 & -9.86 \\
Pr III & & -9.62 & -11.32 \\
Nd III & & $-9.57 \pm 0.08$ & -10.62 \\
Yb II & -8.10 & $-8.10 \pm 0.19$ & -11.20 \\
Yb III & $>-8.10$ & $-7.31 \pm 0.01$ & -11.20 \\
Os II & & -10.55 & -10.64 \\
Ir II & -11.15 & $-10.66:$ & -10.66 \\
Pt & -10.42 & -7.63 & -10.42 \\
Au II & -7.51 & $-7.51 \pm 0.06$ & -11.12 \\
Au III & -7.51 & & -11.12 \\
Hg I & -6.60 & $-6.19 \pm 0.18$ & -10.87 \\
Hg II & -6.60 & $-6.53 \pm 0.23$ & -10.87 \\
Hg III & -6.60 & & -10.87 \\
\hline
\end{tabular}

interval. We used the ATLAS12 model atmosphere adopted in Castelli \& Hubrig (2004) and the abundances listed in the second column of Table 1 . We started with the abundances adopted to compute the optical spectrum and then we modified some of them in order to better fit the ultraviolet spectrum. The abundances of $\mathrm{B}, \mathrm{N}, \mathrm{Al}, \mathrm{Cl}, \mathrm{V}, \mathrm{Zn}, \mathrm{Ge}, \mathrm{As}, \mathrm{Ag}$, and $\mathrm{Cd}$ were derived only in this paper. For those elements that do not show lines in the HST-STIS spectrum we adopted the abundances derived from the optical region if they did not produce inconsistent results, as is the case of Pt. For the ions C I, P I, Ga III, Y III, and $\mathrm{Yb}$ III the ionization equilibria are not satisfied. We did not use their corresponding abundance to compute the synthetic spectrum, but that of the ions in the other ionization stages. In addition, the abundance derived from Mn I and Mn II gives incorrect profiles for a large number of Mn III lines, that are either not observed or are computed as too strong (Fig. 1). These lines are mostly due to transitions having either an even level with energy larger than $172000 \mathrm{~cm}^{-1}$ or an odd level with energy larger than $130000 \mathrm{~cm}^{-1}$. We compared the Kurucz log $g f$ values that we used with those from Uylings \& Raassen (1997) for the lines in common. Because the differences are not larger than 0.2 dex (while fitting the observed lines requires lowering the $\log g f$ values by 1.0 up to 2.0 dex or more), we could argue that some physical mechanism like the vertical abundance stratification weakens the high-excitation lines of Mn III in HD 175640. On the other hand, the low-excitation lines, in particular those of multiplets 10, 11, 12, 13, 14, 15, 16, 17, and 18 (Moore 1950), which lie in the $1900-2400 \AA$ region, are reasonably well predicted by the adopted abundance.

To compute the synthetic spectrum we used the Kurucz database which is formed by the Kurucz (1988; 1993) line lists and by new and revised data (Kurucz 2011). The Kurucz line lists include the hyperfine structure for several elements. In particular, for Mn II, the magnetic dipole and electric quadrupole coupling constants A and B measured by Holt et al. (1999) for 59 levels were used to compute the hyperfine structure of all the lines with coupling constants available for both levels. The

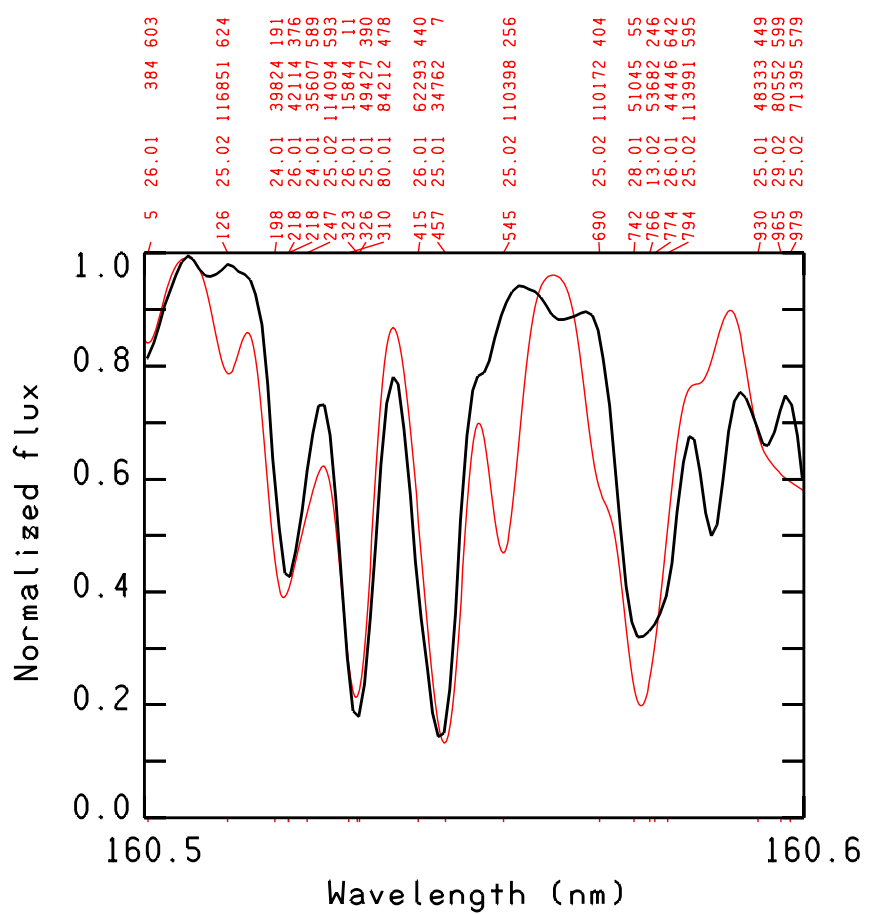

Fig. 1. Example of computed spectrum (red line) displaying Mn III lines not observed in the stellar spectrum (black line). The lines are those at $1605.126 \AA, 1605.545 \AA$, and $1605.690 \AA$. Their upper level is an even level with energy $179152.090 \mathrm{~cm}^{-1}, 172682.640 \mathrm{~cm}^{-1}$, and $172451.230 \mathrm{~cm}^{-1}$, respectively. The labels for the lines include the last three digits of the wavelength, the code (atomic number) for the element and ion, the lower energy level in $\mathrm{cm}^{-1}$, and the residual intensity at line center in per mil.

constants and the hyperfine lines are listed on Kurucz's website ${ }^{1}$. The two files quoted in the footnote differ in that experimental $\log g f$ values, when available, are used in the second file instead of the Kurucz computed values which are stored in the first file.

For this work we added lines of Ga II, Ga III, Rh II, Pd II, Ag II, Y III, Zr III, Yb III, Pt II, Pt III, Au II, Au III, Hg II, Hg III. The presence of these elements in the STIS spectrum of HD 175640 was inferred from the previous analysis of the optical region (Castelli \& Hubrig 2004). We also used predicted lines and intensities for the STIS region using the "predicted stellar" option of the VALD database (Ryabchikova et al. 1997) with stellar parameters for HD 175640 from Castelli \& Hubrig (2004). Additionally, we used results from wavelength coincidence statistic (WCS, Cowley \& Hensberge 1981), using measurements of STIS wavelengths for $\lambda>2000 \AA$. These wavelengths are available online ${ }^{2}$. More information on the heavy elements identified in HD 175640 is given in the Appendix.

In addition to the large overabundance of $\mathrm{Mn}([+2.4])$, $\mathrm{Ti}([+1.4]), \mathrm{Cr}([+1.0])$, and the mild underabundance of $\mathrm{Fe}([-0.3])$, mild overabundances of $\mathrm{B}, \mathrm{Na}$, and $\mathrm{P}$, a general underabundance of the light elements (He, C, N, Mg, Al, Si, S, $\mathrm{Cl})$, and general overabundance of several heavy elements $(\mathrm{Cu}$, $\mathrm{Ga}, \mathrm{As}, \mathrm{Br}, \mathrm{Sr}, \mathrm{Y}, \mathrm{Zr}, \mathrm{Rh}, \mathrm{Pd}, \mathrm{Ag}, \mathrm{Cd}, \mathrm{Xe}, \mathrm{Yb}, \mathrm{Au}$, and $\mathrm{Hg}$ ) were measured. This peculiar chemical composition gives rise to a very rich line spectrum in the ultraviolet.

\footnotetext{
1 http://kurucz.harvard.edu/atoms/hyper250155.pos or http://kurucz . harvard.edu/atoms/hyper250155.all

2 http://dept.astro.1sa.umich.edu/ cowley/HR7143air. html
} 


\section{New Mn II energy levels}

After adding to the Kurucz line list the missing atomic species relevant for the star and available in the literature, and after comparing the observed spectrum with the synthetic spectrum computed with the abundances given in Table 1, we concentrated on the Mn II lines. Line data for this ion were recently updated and critically evaluated by Kramida \& Sansonetti (2013). They list $3969 \mathrm{Mn}$ II lines from $933.0682 \AA$ to $9907.26 \AA$ from which they derived 277 even energy levels and 377 odd energy levels. All the lines are classified as transitions between known energy levels, except for 188 lines observed in the laboratory spectra but not classified. Most of these lines lie in the $2380-2700 \AA$ region. Numerous unidentified lines can be observed in this region in HD 175640. The wavelengths of several of them are the unclassified Mn II lines.

To determine new energy levels we used the same method adopted by Castelli \& Kurucz (2010) when they derived new Fe II energy levels from the optical UVES spectrum of HR 6000. Predicted energy levels and $\log g f$ values for Mn II were computed by Kurucz with his version of the Cowan (1981) code (Kurucz 2011). The calculation included 50 even configurations $d^{6}, d^{5} 4 d-12 d, d^{4} 4 s 4 d-4 s 9 d, d^{5} 4 s-12 s, d^{4} 4 s^{2}$, $d^{4} 4 s 5 s-4 s 9 s, d^{5} 5 g-9 g, d^{4} 4 s 5 g-4 s 9 g, d^{5} 7 i-9 i, d^{4} 4 s 7 i-4 s 9 i$, $d^{4} 4 s 91$, and $d^{4} 4 p^{2}$ with 19686 eigenvalues that were fitted with the least-squares method to 277 known levels. The 41 odd configurations included $d^{5} 4 p-10 p, d^{4} 4 s 4 p-4 s 10 p, d^{3} s^{2} 4 p$, $d^{5} 4 f-10 f, d^{4} 4 s 4 f-4 s 10 f, d^{5} 6 h-9 h, d^{4} 4 s 6 h-4 s 9 h, d^{5} 8 k-9 k$, and $d^{4} 4$ s8k-4s9k with 19820 eigenvalues which were least-squares fitted to 377 known levels. The calculations were done in intermediate coupling with all configuration interactions included, with scaled Hartree-Fock starting guesses, and with HartreeFock transition integrals. A total of 5146779 lines were saved from the transition array of which 41882 lines are between known levels and have good wavelengths.

To derive new energy levels for Mn II, we considered predicted wavelengths due to transitions between a measured and a predicted energy level. A predicted line is usually not shifted more than $\pm 10 \AA$ from the corresponding unknown observed line. However, owing to the large number of predicted lines, of unidentified lines, and blends in the ultraviolet spectrum of HD 175640, it is not a straightforward task to find the correct correspondence between a predicted line and an unidentified line observed in the spectrum.

The computed line list was sorted into tables of all the lines with $\log g f \geq-3.0$ connected to every predicted level. At first, we extracted the lines with $\log g f \geq-1.0$ so that only the strongest lines were examined. When at least two strong predicted lines originate from the same predicted level, we searched in the spectrum for an unidentified line with wavelength close to the first of the two predicted lines. From the observed wavelength and the known energy level involved in the transition we derived a possible value for the unknown energy level. We used this energy value to derive the corresponding wavelength of the second predicted line. If there is an unidentified line at this position in the observed spectrum we checked the energy value on other predicted lines connected to the examined predicted level. If the test is positive for a sample of lines (usually from 3 to 5), we assign the checked energy to the unknown level. Otherwise, we selected some other observed unidentified line in the spectrum and we repeated the procedure. We proceed in this way until we find that value for the unknown energy that produces lines which are all observed in the spectrum, but not identified.
Whenever one or more new levels was found, the whole semi-empirical calculation was repeated to produce improved predicted wavelengths and $\log g f$ values.

Using the above procedure we identified 58 new energy levels from the HST-STIS spectrum of HD 175640; five of them have odd parity, the others have even parity. We added 15 more new odd parity energy levels that we obtained from the UVES spectrum of HD 175640. The new energy levels are listed in Tables 2 and 3.

The search for new energy levels in the optical region was rather unsuccessful. The energies $98423.93 \mathrm{~cm}^{-1}$ and $98424.00 \mathrm{~cm}^{-1}$ of the two levels belonging to the term $3 d^{5}\left({ }^{6} S\right) 4 f^{7} F^{o}$, were obtained from the strong lines of the triplets at $5294 \AA$ and $5295 \AA$, which are part of the multiplet $3 d^{5}\left({ }^{6} S\right) 4 d e^{7} D^{0}-3 d^{5}\left({ }^{6} S\right) 4 f^{7} F^{o}$. All the transitions of this multiplet give rise to five strong Mn II lines observed at 5294, 5295, 5297, 5299, and $5302 \AA$. Because the lines computed with the energies listed by Kramida \& Sansonetti (2013) are more or less displaced from the observed lines, we modified the energy of the upper level in order to better fit the observed line position. Table 4 compares the energies of the $3 d^{5}\left({ }^{6} \mathrm{~S}\right) 4 \mathrm{f}^{7} \mathrm{~F}^{\mathrm{o}}$ term adopted in this paper with those listed in the NIST database. The corresponding wavelengths of the multiplet are also listed. Figure 2 shows the five Mn II lines computed with wavelengths from this paper listed in the first column of Table 4 and those from Kramida \& Sansonetti (2013) listed in the last column of Table 4.

The energies $113840.6 \mathrm{~cm}^{-1}, \quad 113840.7 \mathrm{~cm}^{-1}$, and $113840.8 \mathrm{~cm}^{-1}$ of the three levels with $J=2$, and 0 respectively, belonging to the odd parity term $3 d^{5}\left({ }^{6} \mathrm{~S}\right) 6 \mathrm{f}^{7} \mathrm{~F}^{\mathrm{o}}$ were simply extrapolated from the levels with higher $J$ quantum number. The transitions from these levels contribute to two weak and blended lines observed at 7166.4 and $7167.4 \AA$.

The energy $119197.79 \mathrm{~cm}^{-1}$ was assumed for all the levels with $J$ from 0 to 7 of the term $3 \mathrm{~d}^{5}\left({ }^{6} \mathrm{~S}\right) 8 \mathrm{f}^{7} \mathrm{~F}^{\mathrm{o}}$. They give rise to two weak lines observed at 5180.271 $\AA$ and 5181.649 $\AA$. The first one is blended with Fe II 5180.312 $\mathrm{A}$.

Finally, the observed lines arising from the three last energy levels of Table 3 are listed in Table A.1 (available at the CDS). In addition to the corrections of the odd parity levels shown in Table 4, we modified two other odd parity energy levels from Kramida \& Sansonetti (2013) on the basis of all the transitions observed in the spectrum related to them. They are: $81802.746 \mathrm{~cm}^{-1}$ instead of $81803.31 \mathrm{~cm}^{-1}\left(3 \mathrm{~d}^{5}\left({ }^{2} \mathrm{I}\right) 4 \mathrm{p} \mathrm{z}^{1} \mathrm{I}^{\mathrm{O}}\right.$, $J=6)$ and $94230.94 \mathrm{~cm}^{-1}$ instead of $94231.20 \mathrm{~cm}^{-1}$ $\left(3 \mathrm{~d}^{5}\left({ }^{2} \mathrm{~S}\right) 4 \mathrm{p} \mathrm{z} \mathrm{z}^{3}, J=2\right)$.

\section{New Mn II lines}

The new Mn II lines due to transitions from the new Mn II energy levels listed in Tables 2 and 3 are given in Table A.1. The new Mn II lines are mostly concentrated in the 2380-2700 ̊ interval. The upper energy levels (Cols. 1-4) were derived as described in Sect. 4; the lower energy levels (Cols. 5-8) were taken from Kramida \& Sansonetti (2013); the wavelength given in Col. 9 is the Ritz wavelength in air for $\lambda \geq 2000 \AA$, in vacuum for $\lambda<2000 \AA$. The $\log g f$ values (Col. 10) were computed by Kurucz with the semi-empirical method, the wavelengths given in Col. 11 are the observed laboratory wavelengths listed by Kramida \& Sansonetti (2013). In addition to the 109 newly classified lines, we give a different assignment for 24 other lines of the Kramida \& Sansonetti (2013) tabulation. This implies different energy levels for these 24 lines. The last column of Table A.1 
F. Castelli et al.: New Mn II energy levels from the STIS-HST spectrum of the HgMn star HD 175640

Table 2. New energy levels of Mn II from the STIS-HST spectrum.

\begin{tabular}{|c|c|c|c|}
\hline \multicolumn{2}{|l|}{ Designation } & \multirow{2}{*}{$\begin{array}{c}J \\
3.0\end{array}$} & \multirow{2}{*}{$\begin{array}{c}\begin{array}{c}\text { Energy } \\
\mathrm{cm}^{-1}\end{array} \\
82303.415\end{array}$} \\
\hline $\begin{array}{l}3 d^{4}\left({ }^{5} D\right) 4 s 4 p\left({ }^{3} P\right) \\
3 d^{4}\left({ }^{5} D\right) 4 s 4 p\left({ }^{3} P\right)\end{array}$ & $\begin{array}{l}{ }^{7} \mathrm{~F}^{\mathrm{o}} \\
{ }^{7} \mathrm{~F}^{\mathrm{o}}\end{array}$ & & \\
\hline $3 d^{5}\left(b^{2} D\right) 4 p$ & ${ }^{3} \mathrm{~F}^{\mathrm{o}}$ & $\begin{array}{l}2.0 \\
4.0\end{array}$ & $\begin{array}{l}100320.586 \\
100725.176\end{array}$ \\
\hline $3 d^{5}\left(b^{2} D\right) 4 p$ & ${ }^{3} \mathrm{P}^{\mathrm{o}}$ & 1.0 & 101807.469 \\
\hline $3 d^{6}\left({ }^{4} G\right) 4 d$ & ${ }^{5} \mathrm{~F}$ & $\begin{array}{l}1.0 \\
2.0 \\
3.0 \\
4.0 \\
5.0\end{array}$ & $\begin{array}{l}106351.582 \\
106348.454 \\
106340.485 \\
106324.758 \\
106298.960\end{array}$ \\
\hline $3 d^{5}\left({ }^{4} G\right) 4 d$ & ${ }^{5} \mathrm{G}$ & $\begin{array}{l}2.0 \\
3.0 \\
4.0 \\
5.0 \\
6.0\end{array}$ & $\begin{array}{l}106408.011 \\
106408.348 \\
106406.642 \\
106400.766 \\
106387.310\end{array}$ \\
\hline $3 d^{5}\left({ }^{4} G\right) 4 d$ & ${ }^{3} \mathrm{D}$ & 3.0 & 107192.929 \\
\hline $3 d^{5}\left({ }^{4} G\right) 4 d$ & ${ }^{3} I$ & $\begin{array}{l}5.0 \\
6.0 \\
7.0\end{array}$ & $\begin{array}{l}107155.877 \\
107145038 \\
107114.165\end{array}$ \\
\hline $3 d^{5}\left({ }^{4} G\right) 4 d$ & ${ }^{3} \mathrm{G}$ & $\begin{array}{l}3.0 \\
4.0 \\
5.0\end{array}$ & $\begin{array}{l}107999.208 \\
108007.750 \\
108006.293\end{array}$ \\
\hline $3 d^{5}\left({ }^{4} G\right) 4 d$ & ${ }^{3} \mathrm{~F}$ & $\begin{array}{l}2.0 \\
3.0 \\
4.0\end{array}$ & $\begin{array}{l}108420.629 \\
108425.105 \\
108420.479\end{array}$ \\
\hline $3 d^{6}\left({ }^{4} P\right) 4 d$ & ${ }^{5} \mathrm{P}$ & $\begin{array}{l}1.0 \\
3.0\end{array}$ & $\begin{array}{l}108617.023 \\
108399.148\end{array}$ \\
\hline $3 d^{6}\left({ }^{4} P\right) 4 d$ & ${ }^{5} \mathrm{~F}$ & $\begin{array}{l}1.0 \\
2.0 \\
3.0 \\
4.0 \\
5.0\end{array}$ & $\begin{array}{l}108511.206 \\
108521.377 \\
108561.169 \\
108604.283 \\
108661.385\end{array}$ \\
\hline $3 d^{5}\left({ }^{4} G\right) 4 d$ & ${ }^{3} \mathrm{H}$ & $\begin{array}{l}4.0 \\
5.0 \\
6.0\end{array}$ & $\begin{array}{l}108907.450 \\
108906.437 \\
108896.070\end{array}$ \\
\hline $3 d^{6}\left({ }^{4} P\right) 4 d$ & ${ }^{3} \mathrm{D}$ & $\begin{array}{l}1.0 \\
2.0 \\
3.0\end{array}$ & $\begin{array}{l}109709.489 \\
109572.155 \\
109370.762\end{array}$ \\
\hline $3 d^{5}\left({ }^{4} \mathrm{P}\right) 4 \mathrm{~d}$ & ${ }^{3} \mathrm{~F}$ & $\begin{array}{l}2.0 \\
3.0\end{array}$ & $\begin{array}{l}110664.620 \\
110611.014\end{array}$ \\
\hline $3 d^{5}\left({ }^{4} P\right) 4 d$ & ${ }^{3} \mathrm{P}$ & 2.0 & 110998.198 \\
\hline $3 d^{5}\left({ }^{4} D\right) 4 d$ & ${ }^{5} \mathrm{G}$ & $\begin{array}{l}2.0 \\
3.0 \\
4.0 \\
5.0 \\
6.0\end{array}$ & $\begin{array}{l}111744.523 \\
111753.977 \\
111761.546 \\
111761.008 \\
111741.583\end{array}$ \\
\hline $3 d^{5}\left({ }^{4} D\right) 4 d$ & ${ }^{5} \mathrm{P}$ & 3.0 & 111831.748 \\
\hline
\end{tabular}

Table 2. continued.

\begin{tabular}{cccc}
\hline \hline Designation & $J$ & $\begin{array}{c}\text { Energy } \\
\mathrm{cm}^{-1}\end{array}$ \\
\hline $3 \mathrm{~d}^{5}\left({ }^{4} \mathrm{D}\right) 4 \mathrm{~d}$ & ${ }^{5} \mathrm{~S}$ & 2.0 & 111991.133 \\
$3 \mathrm{~d}^{5}\left({ }^{4} \mathrm{D}\right) 4 \mathrm{~d}$ & ${ }^{5} \mathrm{~F}$ & 2.0 & 112141.748 \\
& & 3.0 & 112106.537 \\
& & 4.0 & 112046.403 \\
& & 5.0 & 111943.809 \\
$3 \mathrm{~d}^{5}\left({ }^{4} \mathrm{D}\right) 4 \mathrm{~d}$ & ${ }^{5} \mathrm{D}$ & 4.0 & 113199.572 \\
& & & \\
$3 \mathrm{~d}^{5}\left({ }^{2} \mathrm{I}\right) 4 \mathrm{~d}$ & ${ }^{3} \mathrm{I}$ & 5.0 & 118544.936 \\
& & 6.0 & 118574.338 \\
& & 7.0 & 118585.339 \\
$3 \mathrm{~d}^{5}\left({ }^{2} \mathrm{I}\right) 4 \mathrm{~d}$ & ${ }^{1} \mathrm{~K}$ & 7.0 & 119152.797 \\
$3 \mathrm{~d}^{5}\left({ }^{2} \mathrm{I}\right) 4 \mathrm{~d}$ & ${ }^{1} \mathrm{G}$ & 4.0 & 119937.245 \\
\hline
\end{tabular}

Table 3. New energy levels of Mn II from the optical region (UVES spectrum).

\begin{tabular}{cccc}
\hline \hline Designation & $J$ & $\begin{array}{c}\text { Energy } \\
\mathrm{cm}^{-1}\end{array}$ \\
\hline $3 \mathrm{~d}^{5}\left({ }^{6} \mathrm{~S}\right) 4 \mathrm{f}$ & ${ }^{7} \mathrm{~F}^{\mathrm{o}}$ & 1.0 & 98423.93 \\
& & 0.0 & 98424.00 \\
$3 \mathrm{~d}^{5}\left({ }^{6} \mathrm{~S}\right) 6 \mathrm{f}$ & ${ }^{7} \mathrm{~F}^{\mathrm{o}}$ & 2.0 & 113840.6 \\
& & 1.0 & 113840.7 \\
& & 0.0 & 113840.8 \\
$3 \mathrm{~d}^{5}\left({ }^{6} \mathrm{~S}\right) 8 \mathrm{f}$ & ${ }^{7} \mathrm{~F}^{\mathrm{o}}$ & 6.0 & 119197.79 \\
& & 5.0 & 119197.79 \\
& & 4.0 & 119197.79 \\
& & 3.0 & 119197.79 \\
& & 1.0 & 119197.79 \\
& & 0.0 & 119197.79 \\
$3 \mathrm{~d}^{5}\left({ }^{4} \mathrm{G}\right) 4 \mathrm{f}$ & ${ }^{5} \mathrm{H}^{\mathrm{o}}$ & 7.0 & 125218.640 \\
& & 9.0 & 125243.338 \\
$3 \mathrm{~d}^{5}\left({ }^{4} \mathrm{G}\right) 4 \mathrm{f}$ & ${ }^{5} \mathrm{I}^{\mathrm{O}}$ & 6.0 & 125257.344 \\
\hline
\end{tabular}

indicates whether the new line is listed in the NIST database without any classification (unassigned), listed with a different classification (DIFFERENT assign.), or not listed at all.

Figure 3 shows the Mn II spectrum in the regions 2383-2390 ̊ interval computed before and after the determination of both new energy levels and new Mn II lines. Figure 4 compares the observed spectrum of HD 175640 with the synthetic spectrum computed both with and without the new Mn II lines. The improvement of the comparison between the observed and computed spectra due to the addition of the new Mn II lines is evident.

\section{Mn II log gf values and manganese abundance}

For Mn II, Kramida \& Sansonetti (2013) tabulate in the NIST database the experimental log $g f$ values from Den Hartog et al. (2011), Kling \& Griesmann (2000), and Kling, Schnabel \& Griesmann (2001). For lines with upper levels $3 d^{5}\left({ }^{6} S\right) 4 p z^{5} \mathrm{P}^{o}$ 


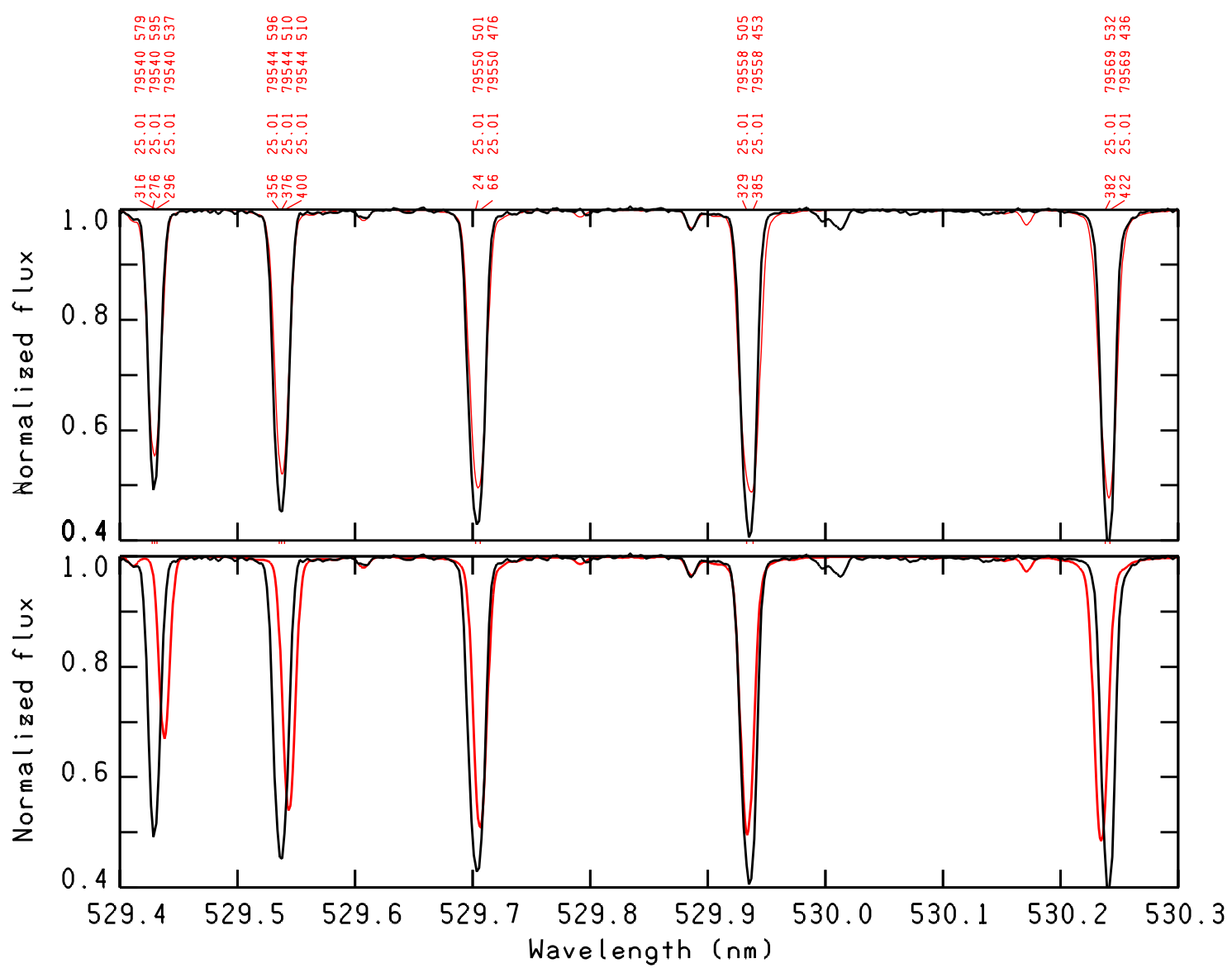

Fig. 2. Improved energy levels of Mn II. Five lines from multiplet $3 d^{5}\left({ }^{6} S\right) 4 d e^{7} D-3 d^{5}\left({ }^{6} S\right) 4 f e^{7} F^{o}$ are computed using the wavelengths and the energies from Kramida \& Sansonetti (2013) (bottom panel), and the current values (upper panel). The new and old wavelengths and energies are listed in Table 4. The meaning of the labels is the same as in Fig. 1.

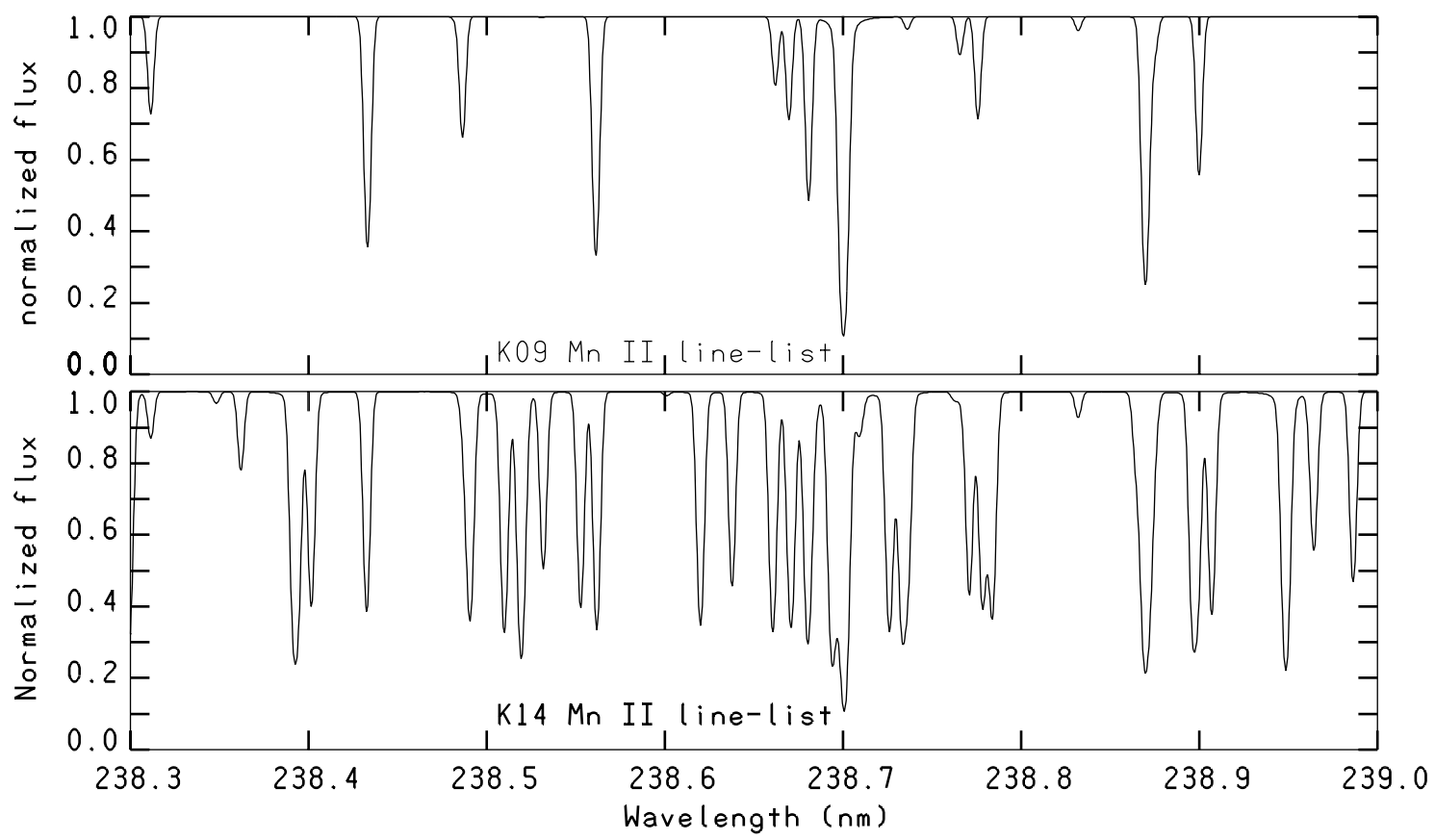

Fig. 3. Upper panel: Mn II synthetic spectrum for the parameters of $\mathrm{HD} 175640\left(T_{\mathrm{eff}}=12000 \mathrm{~K}, \log g=3.95, v \sin i=2.5 \mathrm{~km}{ }^{-1}\right.$, $\left.\left.[\mathrm{Mn} / \mathrm{H}]\right]=+2.4\right)$ computed with the Mn II line list computed by Kurucz before this work (2009 line list); lower panel: the same, but with the new Mn II lines added in the line list. 


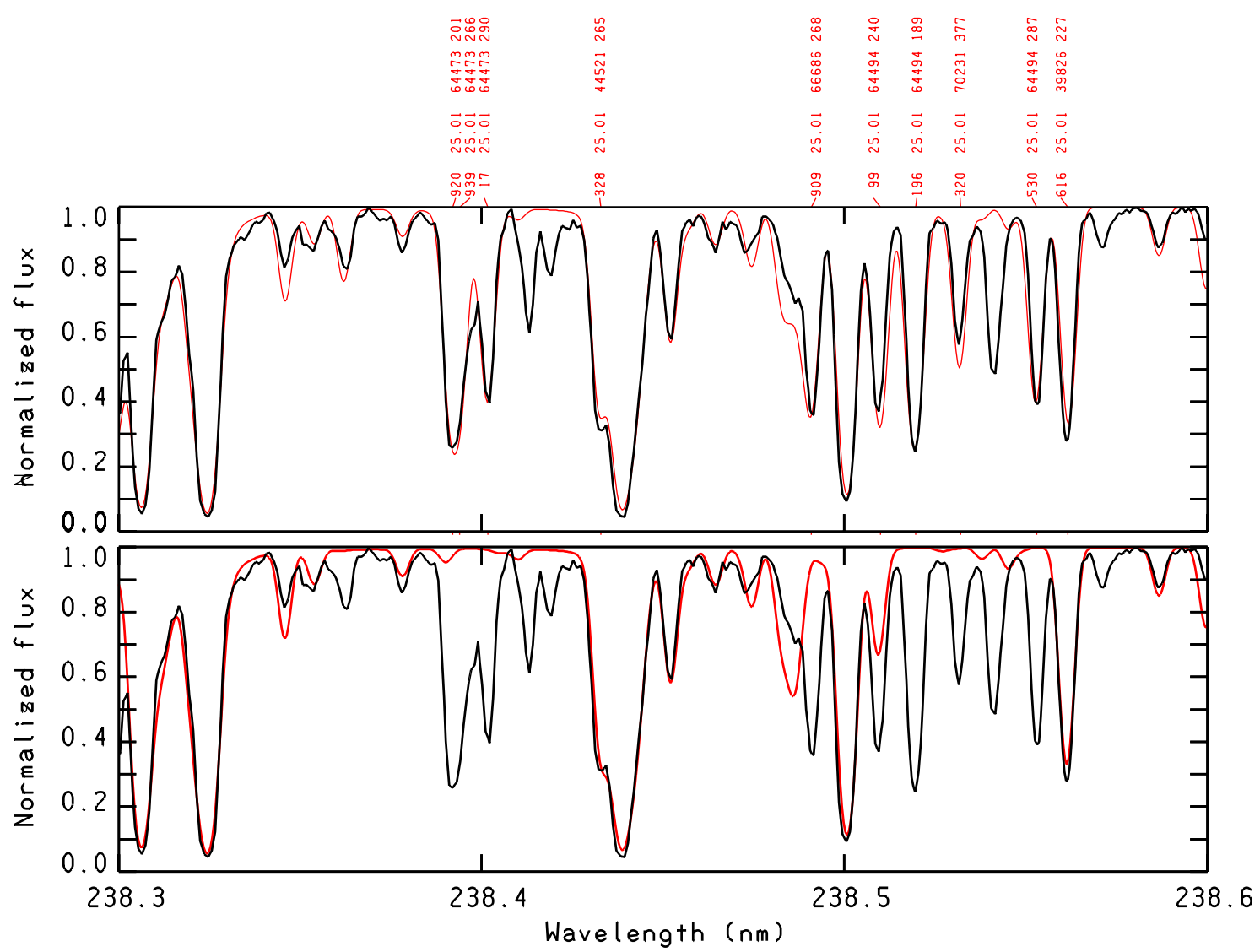

Fig. 4. Upper panel: comparison of the STIS spectrum of HD 175640 (black line) with a synthetic spectrum (red line) computed with a line list including the new Mn II lines; lower panel: the same, but the synthetic spectrum is computed with a line list not including the lines given in Table A.1 (available at the CDS). Only the Mn II lines are indicated with red labels in the plot. The meaning of the labels is the same as in Fig. 1.

Table 4. Multiplet $3 \mathrm{~d}^{5}\left({ }^{6} \mathrm{~S}\right) 4 \mathrm{~d} \mathrm{e}^{7} \mathrm{D}-3 \mathrm{~d}^{5}\left({ }^{6} \mathrm{~S}\right) 4 \mathrm{fe}^{7} \mathrm{~F}^{\mathrm{o}}$.

\begin{tabular}{cccccr}
\hline \hline $\begin{array}{c}\lambda(\mathrm{CKC})^{a} \\
\AA\end{array}$ & $\begin{array}{c}\mathrm{El}(\mathrm{KS})^{b} \\
\mathrm{~cm}^{-1}\end{array}$ & $\begin{array}{c}\mathrm{Eu}(\mathrm{CKC})^{a} \\
\mathrm{~cm}^{-1}\end{array}$ & $\mathrm{Jl}, \mathrm{Ju}$ & $\begin{array}{c}\mathrm{Eu}(\mathrm{KS})^{b} \\
\mathrm{~cm}^{-1}\end{array}$ & $\begin{array}{c}\lambda(\mathrm{KS})^{b} \\
\AA\end{array}$ \\
\hline 5294.276 & 79540.93 & 98424.00 & 1,0 & - & - \\
5294.296 & 79540.93 & 98423.93 & 1,1 & - & - \\
5294.318 & 79540.93 & 98423.858 & 1,2 & 98423.63 & 5294.379 \\
& & & & & \\
5295.356 & 79544.71 & 98423.93 & 2,1 & & \\
5295.376 & 79544.71 & 98423.858 & 2,2 & 98423.63 & 5295.439 \\
5295.400 & 79544.71 & 98423.773 & 2,3 & 98423.63 & 5295.439 \\
& & & & & \\
5297.003 & 79550.50 & 98423.858 & 3,2 & 98423.63 & 5297.063 \\
5297.024 & 79550.50 & 98423.773 & 3,3 & 98423.63 & 5297.063 \\
5297.068 & 79550.50 & 98423.624 & 3,4 & 98423.60 & 5297.072 \\
& & & & & \\
5299.288 & 79558.56 & 98423.773 & 4,3 & 98423.63 & 5299.327 \\
5299.329 & 79558.56 & 98423.624 & 4,4 & 98423.60 & 5299.336 \\
5299.387 & 79558.56 & 98423.424 & 4,5 & 98423.59 & 5299.338 \\
& & & & & \\
5302.327 & 79569.22 & 98423.624 & 5,4 & 98423.60 & 5302.331 \\
5302.382 & 79569.22 & 98423.424 & 5,5 & 98423.59 & 5302.333 \\
5302.421 & 79569.22 & 98423.281 & 5,6 & 98423.56 & 5302.342 \\
\hline & & & & &
\end{tabular}

Notes. The energies and wavelengths from this paper are compared with those from Kramida \& Sansonetti (2013). We note that with the new levels, all 15 of the possible lines of the ${ }^{7} \mathrm{D}-{ }^{7} \mathrm{~F}$ multiplet are known. ${ }^{(a)}$ CKC: this paper; ${ }^{(b)} \mathrm{KS}$ : Kramida \& Sansonetti (2013).

and $3 \mathrm{~d}^{5}\left({ }^{6} \mathrm{~S}\right) 4 \mathrm{pz}^{7} \mathrm{P}^{\mathrm{o}}$, they adjusted the $\log g f$ values given in the last two papers according to the lifetime value recommended by Den Hartog et al. (2011) for these levels. Furthermore, they adopted the semi-empirical log $g f$ values from Kurucz (1988) for several lines.

The comparison of the experimental $\log g f$ values (KGS) with the last version of the semi-empirical oscillator strengths from Kurucz (2014, hereafter K14) is shown in Fig. 5. The average of the difference $\log g f(\mathrm{~K} 14)-\log g f(\mathrm{KGS})$ is $+0.068 \pm 0.397$. The trend is small, but the dispersion around the zero is rather large. However, on a total of 193 lines, 51 of them have a difference in $\log g f$ less than 0.1 dex.

In order to extract a set of lines well suited to deriving the manganese abundance, we presumed that lines with small differences between experimental and semi-empirical $\log g f$ values could be used to this purpose. Table 5 lists $18 \mathrm{Mn}$ II lines with $\log g f$ values from K14 and from either KGS or Den Hartog et al. (2011) differing less than 0.06 dex. The smallest difference $(0.004$ dex $)$ occurs for the line at $4343.983 \AA$. This line gives an abundance of -3.80 dex. We checked this abundance on the other lines listed in Table 5. Instead of deriving approximately the same abundance from the lines, we found a variety of values ranging from -3.8 dex to -4.2 dex, as is shown in the last column of Table 5. We investigated whether the abundance differences are related to the uncertainty associated with the experimental $\log g f$ values. According to Kramida \& Sansonetti (2013) their accuracy is $\leq 0.009$ dex for the score $\mathrm{A}^{+}$and decreases up to $\leq 0.24 \mathrm{dex}$ for the score $\mathrm{D}$. If we restrict our analysis to the lines in Table 5 with the scores $\mathrm{B}, \mathrm{B}^{+}$and $\mathrm{A}^{+}$, corresponding to uncertainties not greater than $0.04 \mathrm{dex}$, we find that the discrepancies in the abundances are not reduced. Figure 6 shows that 
A\&A 580, A10 (2015)

Table 5. Abundances from Mn II lines with experimental and semi-empirical log $g f$ values that differ less than \pm 0.06 dex.

\begin{tabular}{|c|c|c|c|c|c|c|c|c|c|c|c|c|}
\hline $\begin{array}{c}\text { Wave } \\
\AA \\
\end{array}$ & $\begin{array}{c}\log g f \\
\text { K14 }\end{array}$ & $\begin{array}{c}E(\text { low }) \\
\mathrm{cm}^{-1}\end{array}$ & $J($ low $)$ & Term & $\begin{array}{l}E \text { (up) } \\
\mathrm{cm}^{-1}\end{array}$ & $J($ up $)$ & Term & $\mathrm{hfs}$ & $\begin{array}{c}\log g f \\
\text { KSG }\end{array}$ & Acc. & $\begin{array}{l}\Delta \log g f \\
\text { K14-KSG }\end{array}$ & Abund \\
\hline 1678.643 & -2.681 & 9472.993 & 2.0 & $\left({ }^{6} \mathrm{~S}\right) 4 \mathrm{~s}$ a ${ }^{5} \mathrm{~S}$ & 69044.910 & 2.0 & $\left({ }^{4} \mathrm{P}\right) 4 \mathrm{pz}{ }^{3} \mathrm{P}$ & no & -2.638 & $\mathrm{D}^{+}$ & -0.043 & -4.20 \\
\hline 2535.659 & -0.478 & 27583.590 & 4.0 & $\left({ }^{4} \mathrm{G}\right) 4 \mathrm{~s} \mathrm{a}^{5} \mathrm{G}$ & 67009.217 & 3.0 & $\left({ }^{4} \mathrm{P}\right) 4 \mathrm{pz} \mathrm{z}^{5} \mathrm{D}$ & no & -0.502 & $\mathrm{~B}^{+}$ & +0.024 & -3.80 \\
\hline 2535.977 & -1.024 & 27588.534 & 3.0 & $\left({ }^{4} \mathrm{G}\right) 4 \mathrm{~s} \quad \mathrm{a}^{5} \mathrm{G}$ & 67009.217 & 3.0 & $\left({ }^{4} \mathrm{P}\right) 4 \mathrm{p} \mathrm{z}{ }^{5} \mathrm{D}$ & no & -1.045 & $\mathrm{~B}^{+}$ & +0.021 & -3.80 \\
\hline 2543.457 & -0.119 & 27589.360 & 2.0 & $(4 \mathrm{G}) 4 \mathrm{~s} \quad \mathrm{a}^{5} \mathrm{G}$ & 66894.130 & 1.0 & $\left({ }^{4} \mathrm{G}\right) 4 p z^{5} \mathrm{~F}$ & no & -0.138 & $\mathrm{~B}$ & +0.019 & -4.20 \\
\hline 2557.543 & -0.310 & 27588.534 & 3.0 & $\left({ }^{4} \mathrm{G}\right) 4 \mathrm{~s} \quad \mathrm{a}^{5} \mathrm{G}$ & 66676.833 & 2.0 & $\left({ }^{4} \mathrm{P}\right) 4 \mathrm{p} \mathrm{z}{ }^{5} \mathrm{D}$ & no & -0.346 & $\mathrm{C}^{+}$ & +0.036 & -4.10 \\
\hline 2716.793 & -0.664 & 29889.534 & 3.0 & $\left({ }^{4} \mathrm{P}\right) 4 \mathrm{~s} \quad \mathrm{a}^{5} \mathrm{P}$ & 66686.739 & 3.0 & $\left({ }^{4} G\right) 4 p z^{5} F$ & yes & -0.646 & $\mathrm{~B}^{+}$ & -0.018 & -3.80 \\
\hline 2762.558 & -1.422 & 32857.270 & 3.0 & $\left({ }^{4} \mathrm{D}\right) 4 \mathrm{~s} \quad \mathrm{~b}^{5} \mathrm{D}$ & 69044.910 & 2.0 & $\left({ }^{4} \mathrm{P}\right) 4 p z^{3} \mathrm{P}$ & no & -1.471 & $\mathrm{D}^{+}$ & +0.049 & -4.20 \\
\hline 2933.785 & -1.421 & 32818.440 & 0.0 & $\left({ }^{4} \mathrm{D}\right) 4 \mathrm{~s} \quad \mathrm{~b}^{5} \mathrm{D}$ & 66894.130 & 1.0 & $\left({ }^{4} \mathrm{G}\right) 4 p z^{5} \mathrm{~F}$ & yes & -1.458 & B & +0.037 & -4.20 \\
\hline 2935.362 & -2.047 & 32836.740 & 1.0 & $\left({ }^{4} \mathrm{D}\right) 4 \mathrm{~s} \quad \mathrm{~b}^{5} \mathrm{D}$ & 66894.130 & 1.0 & $\left({ }^{4} \mathrm{G}\right) 4 \mathrm{p} \mathrm{z}{ }^{5} \mathrm{~F}$ & no & -2.002 & $\mathrm{C}^{+}$ & -0.045 & -3.85 \\
\hline 2949.204 & +0.313 & 9472.993 & 2.0 & $\left({ }^{6} \mathrm{~S}\right) 4 \mathrm{~s} \mathrm{a}^{5} \mathrm{~S}$ & 43370.537 & 3.0 & $\left({ }^{6} \mathrm{~S}\right) 4 \mathrm{p} \mathrm{z}{ }^{5} \mathrm{P}$ & no & $+0.253^{a}$ & $\mathrm{~A}^{+}$ & +0.060 & -4.20 \\
\hline 2955.139 & -0.972 & 32857.270 & 3.0 & $\left({ }^{4} \mathrm{D}\right) 4 \mathrm{~s} \quad \mathrm{~b}^{5} \mathrm{D}$ & 66686.739 & 3.0 & $\left({ }^{4} G\right) 4 p z^{5} F$ & no & -0.987 & $\mathrm{~B}^{+}$ & +0.015 & -3.80 \\
\hline 2989.730 & -1.934 & 33248.660 & 4.0 & $\left({ }^{4} \mathrm{G}\right) 4 \mathrm{~s} \quad \mathrm{a}^{3} \mathrm{G}$ & 66686.739 & 3.0 & $\left({ }^{4} \mathrm{G}\right) 4 p z^{5} \mathrm{~F}$ & no & -1.924 & $\mathrm{D}^{+}$ & -0.010 & -3.90 \\
\hline 3050.657 & -0.159 & 36274.620 & 2.0 & $\left({ }^{4} \mathrm{P}\right) 4 \mathrm{~s} \quad \mathrm{~b}^{3} \mathrm{P}$ & 69044.910 & 2.0 & $\left({ }^{4} \mathrm{P}\right) 4 \mathrm{pz}{ }^{3} \mathrm{P}$ & no & -0.206 & $\mathrm{~B}^{+}$ & +0.047 & -4.15 \\
\hline 3146.121 & -2.546 & 34910.770 & 4.0 & $d 6 b^{3} 3 G$ & 66686.739 & 3.0 & $\left({ }^{4} \mathrm{G}\right) 4 p z^{5} \mathrm{~F}$ & no & -2.523 & $\mathrm{D}$ & -0.023 & -4.25 \\
\hline 3204.878 & -1.115 & 37851.490 & 3.0 & d6 $a^{3} 3 \mathrm{D}$ & 69044.910 & 2.0 & $\left({ }^{4} \mathrm{P}\right) 4 \mathrm{p} \mathrm{z}{ }^{3} \mathrm{P}$ & no & -1.097 & $\mathrm{~B}^{+}$ & -0.018 & -4.15 \\
\hline 4206.368 & -1.584 & 43528.661 & 5.0 & $\left({ }^{4} \mathrm{~F}\right) 4 \mathrm{~s} \mathrm{a}{ }^{5} \mathrm{~F}$ & 67295.446 & 4.0 & $\left({ }^{4} \mathrm{P}\right) 4 \mathrm{pz} \mathrm{z}^{5} \mathrm{D}$ & yes & -1.553 & $\mathrm{C}^{+}$ & -0.031 & -4.05 \\
\hline 4292.233 & -1.581 & 43395.395 & 3.0 & $\left({ }^{2} \mathrm{D}\right) 4 \mathrm{~s} \mathrm{c}^{3} \mathrm{D}$ & 66686.739 & 3.0 & $\left({ }^{4} \mathrm{G}\right) 4 p z^{5} \mathrm{~F}$ & yes & -1.544 & $\mathrm{D}^{+}$ & -0.037 & -4.20 \\
\hline 4343.983 & -1.105 & 43528.661 & 5.0 & $\left({ }^{4} \mathrm{~F}\right) 4 \mathrm{~s} \mathrm{a}{ }^{5} \mathrm{~F}$ & 66542.539 & 5.0 & $\left({ }^{4} G\right) 4 p z^{5} F$ & yes & -1.109 & $\mathrm{C}^{+}$ & +0.004 & -3.80 \\
\hline
\end{tabular}

Notes. ${ }^{(a)}$ This value is from Den Hartog et al. (2011).

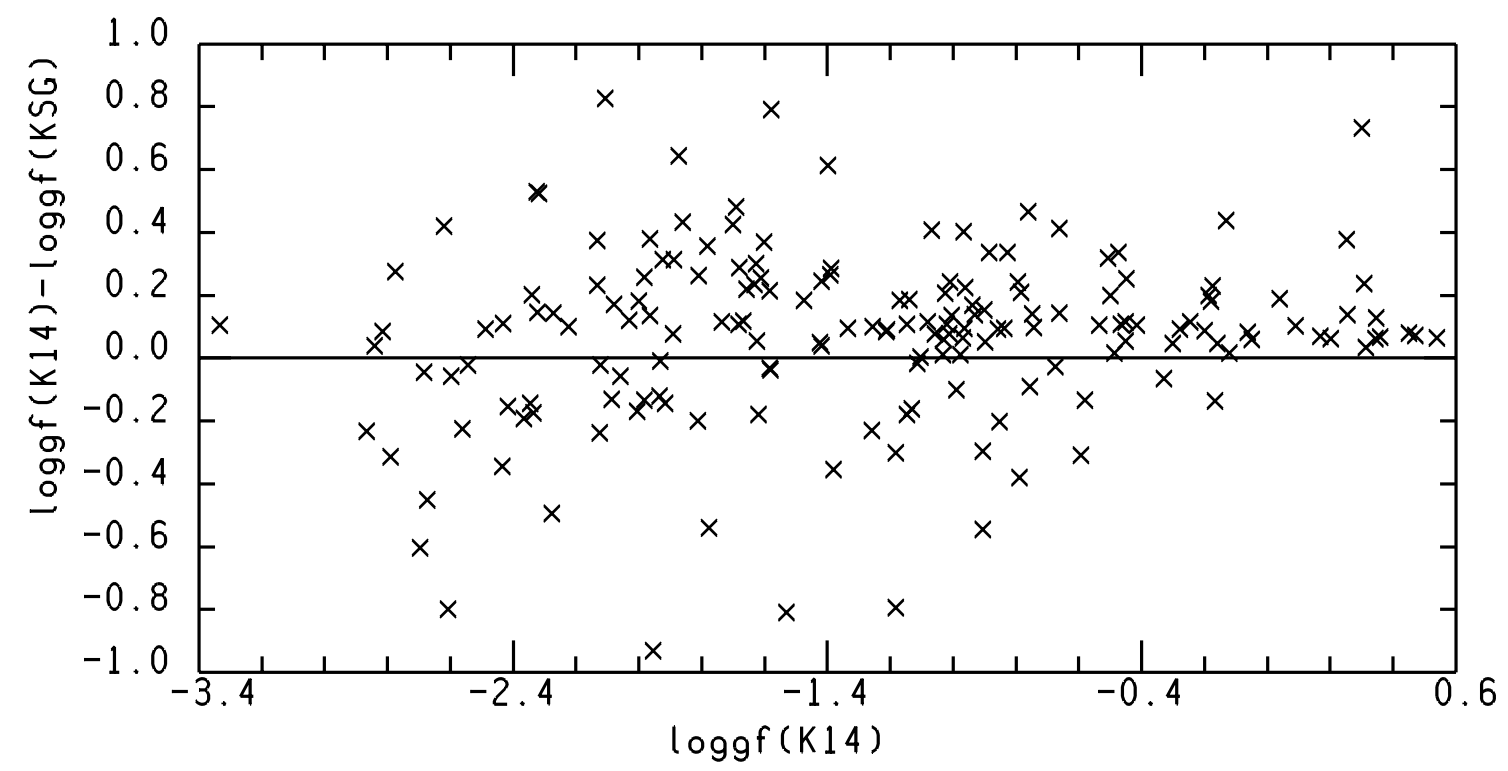

Fig. 5. Comparison of the experimental log $g f$ values from Kling \& Griesmann (2000) and Kling et al. (2001) with the semi-empirical computed values from Kurucz (2014). There are still eight points outside the figure, four with $\log g f>1.0$ and four with $\log g f<-1.0$.

the problem occurs because some strong Mn II lines have profiles that cannot be reproduced by computations for any adopted abundance. For instance, while the high abundance of -3.8 dex improves the agreement between the observed and computed profiles of the strong line with no wings at $2716.793 \AA$, it is too high for the broad line at $2949.204 \AA$, which develops unobserved strong wings for abundances larger than -4.20 dex. We note that the experimental $\log g f$ value of this line is determined with an uncertainty $\leq 0.009 \mathrm{dex}$, so that it cannot be the cause of the weaker than observed computed core for the -4.20 dex abundance, well suited to fitting the wings. An increase in the microturbulent velocity from $0.0 \mathrm{~km} \mathrm{~s}^{-1}$ to higher values does not solve the problem because the effect is the same as that due to an increase in the abundance. The difficulty in fitting the profile of several strong lines can be due to missing hyperfine structure (but this is not the case of the line at $2716.793 \AA$ ); $\log g f$ uncertainties larger than those estimated; the adopted model, which may have an incorrect stratification in the uppermost layers; and some manganese vertical abundance stratification causing a manganese accumulation in the upper layers.

The analysis of all the Mn II lines in HD 175640 from $1250 \AA$ to $10000 \AA$, has shown that the most reliable manganese abundance is that derived from the lines of multiplet 1 in the 3438-3500 ̊ spectral region. The measured equivalent widths listed in Castelli \& Hubrig (2004) together with the log $g f$ values from Den Hartog et al. (2011) give an average abundance of $-4.17 \pm 0.03$ dex. This abundance reproduces very well both the wings and the core of the lines at 3441.896, 3460.315, 3482.904, 

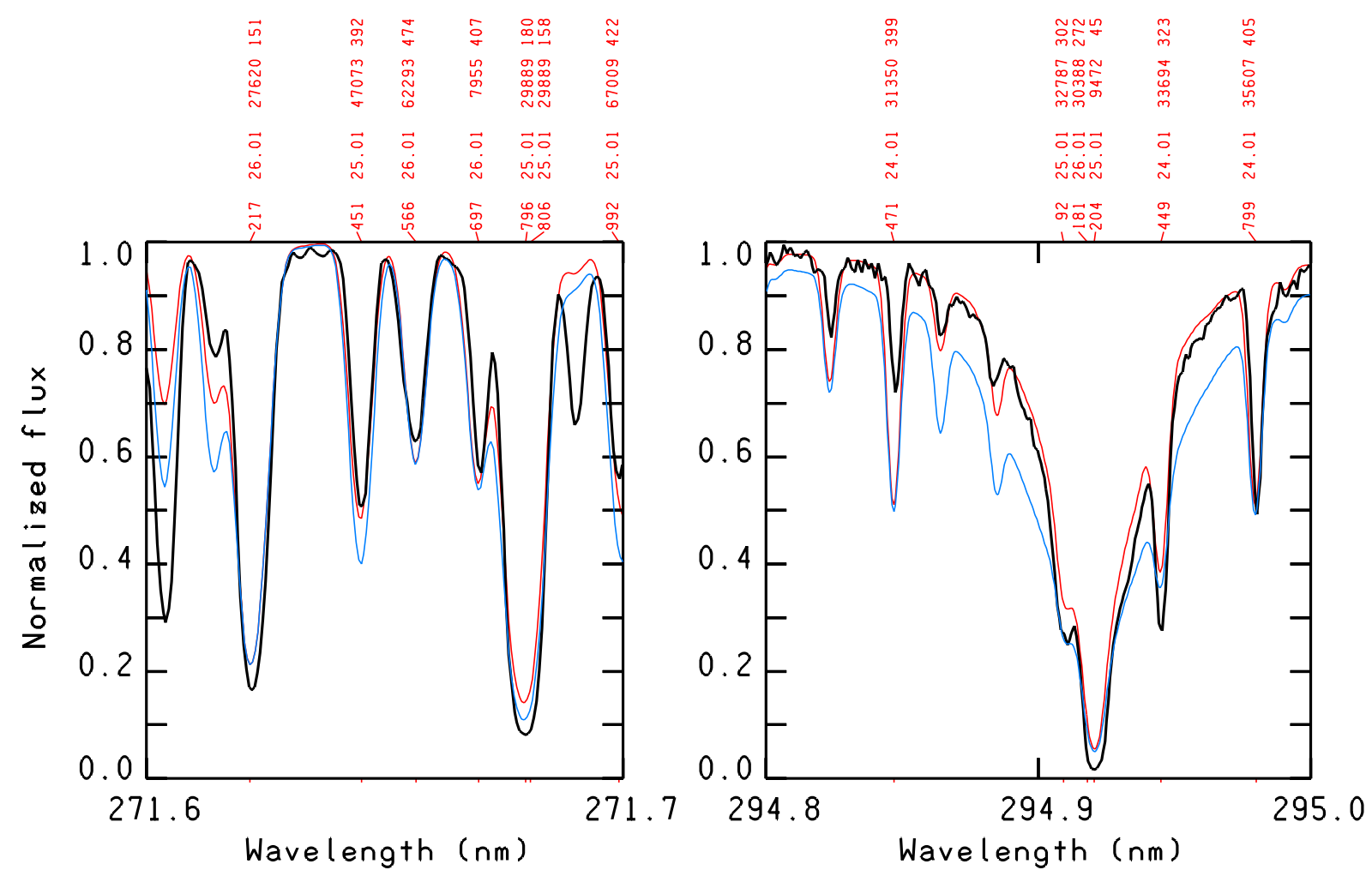

Fig. 6. Comparison of the observed spectrum (black line) with synthetic spectra computed for a manganese abundance of -4.2 dex (red line) and -3.8 dex (blue line). The plot on the left shows how the computed core of the line at $2716.793 \AA$ increases with increasing abundance, although it does not fit the observed spectrum even with -3.8 dex. A still larger abundance would develop unobserved wings. The line is computed with the hyperfine structure included. The strongest hyperfine components are those at $2716.796 \AA$ and $2716.806 \AA$. The plot on the right shows how the wings of the strong Mn II line at $2949.204 \AA$ are well fitted by the profile computed with the abundance of -4.2 dex. The higher abundance gives rise to unobserved wings. The meaning of the labels is the same as in Fig. 1.

Table 6. Mn II lines with experimental and semi-empirical log $g f$ values that differ more than \pm 1.00 dex.

\begin{tabular}{|c|c|c|c|c|c|c|c|c|c|c|c|c|}
\hline $\begin{array}{c}\text { Wave } \\
\AA\end{array}$ & $\begin{array}{c}\log g f \\
\mathrm{~K} 14\end{array}$ & $\begin{array}{c}E \text { (low) } \\
\mathrm{cm}^{-1}\end{array}$ & $J$ (low) & Term & $\begin{array}{l}E \text { (up) } \\
\mathrm{cm}^{-1}\end{array}$ & $J($ up $)$ & Term & $\mathrm{hfs}$ & $\begin{array}{c}\log g f \\
\mathrm{KSG}\end{array}$ & Acc. & $\begin{array}{l}\Delta \log g f \\
\text { K14-KSG }\end{array}$ & $\begin{array}{l}\log g f \text { for the } \\
-4.20 \text { dex abund }\end{array}$ \\
\hline 2559.416 & -0.350 & 27583.590 & 4.0 & $\left({ }^{4} \mathrm{G}\right) 4 \mathrm{sa}^{5} \mathrm{G}$ & 66643.296 & 4.0 & $\left({ }^{4} \mathrm{G}\right) 4 \mathrm{p} \mathrm{z}^{5} \mathrm{~F}$ & no & -1.424 & $\mathrm{D}$ & +1.074 & -0.35 \\
\hline 2717.524 & -0.895 & 29889.534 & 3.0 & $\left({ }^{4} \mathrm{P}\right) 4 \mathrm{~s} \quad \mathrm{a}^{5} \mathrm{P}$ & 66676.833 & 2.0 & $\left({ }^{4} \mathrm{P}\right) 4 \mathrm{p} \mathrm{z}{ }^{5} \mathrm{D}$ & yes & -2.184 & $\mathrm{E}$ & +1.289 & -0.70 \\
\hline 2719.736 & -0.319 & 29919.444 & 2.0 & $\left({ }^{4} \mathrm{P}\right) 4 \mathrm{sa}{ }^{5} \mathrm{P}$ & 66676.833 & 2.0 & $\left({ }^{4} \mathrm{P}\right) 4 \mathrm{p} \mathrm{z}{ }^{5} \mathrm{D}$ & yes & -1.500 & D & +1.181 & -0.319 \\
\hline 2845.848 & -2.732 & 31514.710 & 4.0 & $\mathrm{~d} 6 \mathrm{a}^{3} \mathrm{~F}$ & 66643.296 & 4.0 & $\left({ }^{4} \mathrm{G}\right) 4 p z^{5} \mathrm{~F}$ & no & -1.274 & $\mathrm{D}^{+}$ & -1.458 & -2.85 \\
\hline 2956.005 & -1.325 & 32857.270 & 3.0 & $\left({ }^{4} \mathrm{D}\right) 4 \mathrm{~s} \mathrm{~b}^{5} \mathrm{D}$ & 66676.833 & 2.0 & $\left({ }^{4} \mathrm{P}\right) 4 \mathrm{pz}^{5} \mathrm{D}$ & no & -3.168 & $\mathrm{D}$ & +1.843 & -0.75 \\
\hline 2993.603 & -2.421 & 33147.710 & 5.0 & $\left({ }^{4} \mathrm{G}\right) 4 \mathrm{~s} \quad \mathrm{a}^{3} \mathrm{G}$ & 66542.539 & 5.0 & $\left({ }^{4} \mathrm{G}\right) 4 p z^{5} \mathrm{~F}$ & no & -1.061 & $\mathrm{D}^{+}$ & -1.360 & -2.35 \\
\hline 4325.047 & -2.355 & 43528.661 & 5.0 & $\left({ }^{4} \mathrm{~F}\right) 4 \mathrm{~s} \quad \mathrm{a}^{5} \mathrm{~F}$ & 66643.296 & 4.0 & $\left({ }^{4} \mathrm{G}\right) 4 p z^{5} \mathrm{~F}$ & yes & -1.115 & $\mathrm{C}^{+}$ & -1.240 & -2.55 \\
\hline 4393.385 & -2.646 & 44139.031 & 1.0 & $\left({ }^{2} \mathrm{D}\right) 4 \mathrm{~s}_{\mathrm{c}} \mathrm{c}^{3} \mathrm{D}$ & 66894.130 & 1.0 & $\left({ }^{4} \mathrm{G}\right) 4 p z^{5} \mathrm{~F}$ & yes & -1.458 & $\mathrm{D}^{+}$ & -1.188 & -2.70 \\
\hline
\end{tabular}

and $3488.676 \AA$. For the other lines of the multiplet the observed core is a little stronger than the computed value.

There are eight points outside the borders of Fig. 5, which were not plotted. They correspond to the lines listed in Table 6 for which the difference between the K14 and KSG log $g f$ values is larger than \pm 1.0 dex. The uncertainties of the experimental $\log g f$ values is high because it is included between $\leq 0.08 \mathrm{dex}\left(\right.$ score $\mathrm{C}^{+}$) and $>0.24 \mathrm{dex}$ (score E). We note that the source of the $\log g f$ values for the first three lines in Table 6 was erroneously indicated in the NIST database as c88 (Kurucz 1988) instead of T7259 (Kling et al. 2001). The $\log g f$ value needed to reproduce the line profile when the abundance of -4.20 dex is adopted is given in the last column of Table 6. For all the lines, this $\log g f$ value is closer to that from K14 than to that from KGS. This result suggests that, for some lines, the experimental $\log g f$ values may be less reliable than the calculated values.

\section{Comparison of the observed and computed spectra}

The plot of the comparison of the observed and computed spectra of HD 175640 in the whole $1250-3040 \AA$ region is available at the Castelli website ${ }^{3}$.

When we compare the whole observed region from $1250 \AA$ to $3046 \AA$ with the synthetic spectrum we are far from satisfied. From $1250 \AA$ to $1692 \AA$ the agreement is poor, except for a

\footnotetext{
3 http://wwwuser.oats.inaf.it/castelli/hd175640stis/ tab1250-3040.html
} 
few lines. In fact, in spite of the rather high resolving power ( $R \sim 40000-50000)$, the blends are so numerous and formed by so many components that it is not easy to compute them correctly. There are numerous missing lines, lines computed that are too strong, lines computed that are too weak, and lines probably affected by small wavelength errors. Furthermore, for a few elements, the abundance is different for the different ionization states. Between $1692 \AA$ and $2332 \AA$ the comparison is even worse because all the above problems are increased by the rather low resolving power $(R \sim 25000-30000)$. Only in the 2332-3040 $\AA$ region does the comparison show a rather good agreement. This range is less crowded with lines than the previous regions and was observed with a very high resolving power $(\sim 120000)$.

\subsection{Problematic Mn II lines}

If we restrict the discussion only to the Mn II lines, we see that there are several lines with computed profiles without an observed counterpart. For instance, all the lines arising from the even parity level $43131.51 \mathrm{~cm}^{-1}\left(3 \mathrm{~d}^{6} \mathrm{a}^{1} \mathrm{D}, J=2\right)$ are predicted as being strong, but they are either not observed at all or have a very weak observed counterpart. Another remarkable line is that at $2703.213 \AA$, which is predicted as very strong with both the Kurucz (2014) and Kling et al. (2001) $\log g f$ values, i.e. -0.484 and -0.808 , respectively, but is not observed at all in the spectrum. It would be too long to list here all the Mn II lines computed as too strong compared to the observed ones. In some cases, a possible explanation for the disagreement is the severe mixing affecting one of the two energy levels involved in the transition and the difficulty in computing it. Furthermore, it is very likely that the computed stellar atmosphere is inadequate to explain all the lines observed in the spectrum, in particular for manganese which is very overabundant. In addition to the shortcoming affecting the core of the strong Mn II lines which, as discussed in Sect. 6, is computed as too weak compared to the observed core, there are several Mn II lines in the optical spectrum that seem to be weakened by some emission. While the computed lines are strong the observed lines are weak or even absent. All these lines are due to transitions between high-energy levels like the blend at $6446.188,6446.247,6446.330 \AA$, the lines at $6462.220,6462.465,6462.800,6463.200,6463.627 \AA$, those at $9903.853,9904.428,9905.216,9906.2$, and 9907.2 $\AA$, among others.

On the other hand, there are a few strong lines observed in the spectrum that are identified as Mn II in the NIST database, but which are either not predicted by the Kurucz (2014) $\log g f$ values or are predicted as much too weak. Table A.2 (available at the CDS) lists the most significant lines.

\subsection{Absorption components of resonance lines}

A few strong resonance lines are either double with a red component or are very broad with a redshifted line core. In this last case, the profile is the blend of the line predicted at the Ritz wavelength with an unpredicted redshifted line. In Table 7 we list in Col. 2 the Ritz wavelength and in Cols. 3 and 4 the wavelengths $\lambda_{\text {obs }}$ and $\lambda_{\text {comp }}$ of the two components when they can be disentangled. In this case $\lambda_{\mathrm{obs}}$ is the same as the Ritz wavelength, otherwise $\lambda_{\text {obs }}$ is the wavelength of the blend of the two components unless the line is so saturated that the core lies below the zero level of the normalized flux scale and cannot be measured. The velocity shift of the component is given in Col. 5. We
Table 7. Lines affected by a redshifted component.

\begin{tabular}{lccccl}
\hline \hline Elem & $\lambda($ Ritz $)$ & $\lambda(\mathrm{obs})$ & $\lambda_{\text {comp }}$ & $V_{\text {shift }}$ & Notes \\
\hline Mg I & 2852.126 & 2852.126 & 2852.20 & 7.78 & double \\
Mg II & 2795.528 & & & & saturated \\
& 2802.705 & & & & saturated \\
Si II & 1808.013 & 1808.03 & & & \\
S II & 1250.584 & 1250.60 & & & \\
Mn II & 2576.104 & 2576.104 & 2576.18 & 8.84 & double \\
& 2593.721 & 2593.721 & 2593.80 & 9.13 & double \\
& 2605.680 & 2605.68 & 2605.75 & 8.05 & double \\
Fe II & 2343.495 & 2343.53 & & & \\
& 2373.735 & 2373.735 & 2373.80 & 8.21 & double \\
& 2382.037 & & & & saturated \\
& 2585.876 & 2585.876 & 2585.94 & 7.42 & double \\
& 2599.395 & & & & saturated \\
Zn II & 2025.484 & 2025.51 & & & \\
& 2062.001 & - & 2062.06 & 8.57 & \\
\hline
\end{tabular}

estimate an error of about $2.5 \mathrm{~km} \mathrm{~s}^{-1}$ related to an uncertainty of $0.02 \AA$ in the position of the component on the wavelength scale. The average velocity shift is $8.29 \pm 0.56 \mathrm{~km} \mathrm{~s}^{-1}$. We note that the Zn II line at $2062.001 \AA$ is predicted but not observed, while an unidentified line is present at 2062.060 $\AA$. We assumed that it is the red component of Zn II 2062.001 $\AA$. However, it could be the unpredicted line of some other element as well.

Other similar peculiarities observed in HD 175640 are the bump and the broad weak component affecting the red wing of the Ca II profiles at $3933 \AA$ (K-line) and $3968 \AA$ (H-line), respectively. Furthermore, strong red components can be observed for the Na I lines at $5890 \AA$ and $5896 \AA$ (Castelli \& Hubrig, 2004). We measured a velocity shift for the $\mathrm{Na}$ I components equal to $+7.4 \pm 0.7 \mathrm{~km} \mathrm{~s}^{-1}$ and $+7.7 \pm 0.7 \mathrm{~km} \mathrm{~s}^{-1}$, respectively.

We cannot say whether the redshifted components are of interstellar or circumstellar origin.

\section{Conclusions}

Ultraviolet and optical stellar spectra of the HgMn slowly rotating star HD 175640, observed with both HST-STIS and UVES instruments, were used to extend and discuss the atomic data of Mn II available in the NIST database (Kramida \& Sansonetti 2013). To this purpose, Mn II lines arising both from levels observed in the laboratory and from levels predicted with semiempirical methods by Kurucz, were adopted.

We assigned wavelengths, energy levels and $\log g f$ values to about $257 \mathrm{Mn}$ II lines. Of them, 109 lines had already been identified as Mn II by Kramida \& Sansonetti (2013), but were unclassified. For another 24 lines we assigned different energy levels from those in the NIST database. This implies different energy levels, different $\log g f$ values, and therefore a different intensity for the lines. The new Mn II line data improve the computation of the synthetic spectra of B-type stars, although more work needs to be done on the atomic data, especially in the ultraviolet. For instance, we note that in the NIST database only wavelengths and intensities are listed for the Mn III lines of the 1250-3040 A region analyzed in the paper. No energy levels and no $\log g f$ values are given for them.

As by-product of this study we have extended to the ultraviolet region the abundance analysis performed by Castelli \& Hubrig (2004) in the optical region. Except for cobalt, iridium, 
and platinum, we confirmed the optical abundances within the error limits of the equivalent widths analysis made by Castelli\& Hubrig (2004). Abundances for elements not observed in the visible, such as B, N, Al, Cl, V, Zn, Ge, As, Ag, and Cd were obtained. The abundance pattern is similar to that of other $\mathrm{HgMn}$ stars which have underabundances of the light elements and overabundances of some iron group elements and of some heavy elements. In the case of HD 175640, the most overabundant elements are Ti, Cr, Mn, Ga, As, Br, Y, Zr, Rh, Pd, Ag, Xe, Yb, Au, and $\mathrm{Hg}$.

\section{Appendix A: Heavy elements in HD 175640}

We based the abundance analysis in the ultraviolet mostly on the lines listed by Castelli et al. (1985) and by Castelli \& Bonifacio (1990) in their study of HR 6000 and $\iota$ Her, respectively. The multiplet numbers given in the above papers refer to the Ultraviolet Multiplet Table by Moore (1950). In addition, we made a wide use of the NIST database (Kramida et al. 2014) to search for the most intense lines of a given element. In this section we give some more details only about heavy elements from copper to mercury.

Copper $(\mathrm{Cu}) \mathrm{Z}=29$ : the abundance of -6.50 dex was estimated from the $\mathrm{Cu}$ II lines at 1358.773, 1367.951, 1472.395, 2112.100, 2135.981, 2192.268, 2242.618, and 2247.003. . All the lines are blended. The log $g f$ values are data computed by Kurucz in 2011 and were taken from his database.

Zinc $(\mathrm{Zn}) \mathrm{Z}=30$ : the zinc abundance was derived from the line of $\mathrm{Zn}$ II at $2064.227 \AA$. The $\log g f$ value +0.070 is from the NIST database. The Zn II line at 2062.001 $\AA$ originating from a lower level with energy equal to $0.00 \mathrm{~cm}^{-1}$ is predicted, but not observed. The $\log g f$ value -0.329 is from the Kurucz database. The other line from the zero energy level at $2025.484 \AA$ is computed too weak and is blended with a strong unidentified line that we assumed to be of interstellar or circumstellar origin. No $\mathrm{Zn}$ III lines were observed.

Gallium (Ga) Z = 31: for Ga I, Ga II, and Ga III, wavelengths, energy levels, and $\log g f$ values were taken from the NIST database when available.

For Ga I, only the lines at 2874.23, 2943.64, and 2944.17 were well observed. For the Ga II lines with $\log g f$ values not available in the NIST database, we used $\log g f$ values from Nielsen et al. (2005) (lines at at 1463.576, 1473.690, 1483.453, and $1483.903 \AA$ ). For the remaining lines $\log g f$ values were computed from the lifetime measuraments of Ansbacher et al. (1985) or estimated on the basis of laboratory intensities and excitation energies (Castelli \& Parthasarathy 1995). The abundance -5.43 dex $([+3.6])$ derived from the optical region adequately reproduces the unblended Ga II lines at 1473.690, $1483.903,1504.334,1536.276 \AA$, as well as the blended lines at $1514.505,1535.312 \AA$. The Ga II broad line at $1414.399 \AA$ has too narrow wings, but the classical broadening parameters adopted here are probably not correct. Vice versa, both Ga III profiles at 1495.045 and $1534.462 \AA$ display wings that are too broad. Only these two Ga III lines have $\log g f$ values available in the NIST database. For other lines we adopted estimated values. No isotopic and hyperfine structure was considered in the computations.

Germanium (Ge) $\mathrm{Z}=32$ : several Ge II lines were observed. From the line at $1649.19 \AA$ we derived an underabundance of 1.7 dex relative to the solar value. We adopted $\log g f=-0.28$ from the NIST database.

Arsenic (As) $Z=33$ ): the lines of As II at 1263.77, 1266.34, 1280.987 , and $1287.54 \AA$ were observed in the spectrum. We adopted $\log g f$ values from Warner \& Kirkpatrick (1969) for all them. The observed lines are adequately predicted for an overabundance of -7.50 dex.

Yttrium (Y) Z = 39: for the Y II lines, we used the Kurucz database, which includes hyperfine components for several lines. All the lines are blended, except for the strong line at $2422.18 \AA$. The abundance of -6.66 dex [+3.2] derived from the optical spectrum is well suited to reproducing the observed profile. We added to the line list the Y III lines from Biémont et al. (2011). We modified the two wavelengths $2817.037 \AA$ and $2946.01 \AA$ in $2817.027 \AA$ and $2945.995 \AA$, in order to match the observed spectrum. The Y III lines at 2367.228, 2414.643, 2817.027, and $2945.995 \AA$ were observed. They are strong lines, either unblended or marginally blended. The abundance from Y III is larger than the abundance from Y II by about 1.0 dex.

Zirconium $(\mathrm{Zr}) \mathrm{Z}=40$ : several weak $\mathrm{Zr}$ III lines were observed in the spectrum, but no $\mathrm{Zr}$ II line. A few $\mathrm{Zr}$ III lines are unblended, like that at $2102.283 \AA$. The $\mathrm{Zr}$ III line data from the NIST database were adopted. The Zr III abundance agrees, within the error limits, with the value we obtained from the $\mathrm{Zr}$ II optical lines.

Rhodium ( $\mathrm{Rh}$ ) $\mathrm{Z}=45$ : numerous weak Rh II lines were observed in the spectrum. The line at $1604.45 \AA$ is unblended. Oscillator strengths from Bäckström et al. (2013), Quinet et al. (2012), and Corliss \& Bozman (1962) were used. The wavelengths from the NIST database agree more closely with the stellar wavelengths than the wavelengths adopted by Bäckström et al. (2013). The difference is on the order of $0.02 \AA$. The abundance of $-8.50 \mathrm{dex}$, which was estimated from the UVES spectra on the basis of estimated log $g f$ values, reproduces the ultraviolet lines in a satisfactory way. None of the Rh III lines listed in the NIST database was identified.

Palladium (Pd) Z = 46: numerous Pd II lines were identified. All the lines from the $4 d^{8} 5 s-4 d^{8} 5 p$ transitions and the strongest lines from the $4 d^{8} 5 p-4 d^{8} 6$ s transitions, together with their oscillator strengths, from Quinet (1996) were added in the line lists. Additional lines were taken from Lundberg et al. (1996) and from the NIST database. Unblended lines are those at 2351.347, 2367.966, 2388.310, 2414.7303, 2426.867, 2433.102, 2446.713, 2457.257, 2472.502, 2486.256, 2488.914, 2505.729, 2565.505, 2569.544, 2635.93, and 2658.72 A. None of the Pd I lines listed in the NIST database was identified. For Pd III, only the line at $1782.55 \AA$, which according to the NIST data is the one with the largest intensity in the $1250-3040 \AA$ range, perhaps contributes to a strong blend observed at $1782.6 \AA$.

Silver (Ag) Z = 47: the Ag II lines at 2246.412, 2248.749, 2357.917, and $2411.345 \AA$ were observed. The log $g f$ values from the NIST database were used when available, otherwise we used the Corliss \& Botzman (1962) data, as we did for the line at $2438.325 \AA$, which is computed as too strong.

Cadmium (Cd) Z = 48: the CdII lines at $2144.393 \AA$ and $2265.019 \AA$ were observed and predicted by adopting a 0.8 overabundance over the solar value. The atomic data are from the Kurucz database. The $\log g f$ values are the same as in the NIST database.

Indium (In) $\mathrm{Z}=49$ : the In II line at $1586.331 \AA$, which is predicted as rather strong for solar abundance and $\log g f$ value from the NIST database, is the main component of a complex blend formed by several other lines. We were unable to determine the indium abundance from the blend.

Barium (Ba) $\mathrm{Z}=56$ : the two strongest Ba II lines at $2304.249 \AA$ and $2335.267 \AA$ are heavily blended, and so they cannot be used 
to confirm the abundance derived from the optical region. No other Ba II lines were observed.

Ytterbium ( $\mathrm{Yb}$ ) Z = 70: we adopted the abundance of $-8.10 \mathrm{dex}$ $([+3.0])$ derived from the UVES spectra. We investigated only the $\mathrm{Yb}$ II lines with $\log g f$ available in the NIST database. The lines at 2185.716, 2653.745, and 2750.478 can be observed as single weak lines. Some other lines are weak components of blends. We added to the line list all the $\mathrm{Yb}$ III lines from Biémont et al. (2001) with an upper energy level lower than $100000 \mathrm{~cm}^{-1}$. There are numerous Yb III lines in the spectrum. Their profile is very sharp and would require an abundance about 1 dex larger than that derived from the $\mathrm{Yb}$ II lines in order to agree with the computed profiles. The same behavior for $\mathrm{Yb}$ II and $\mathrm{Yb}$ III abundances was observed in the visible (Castelli \& Hubrig 2004).

Osmium (Os) $\mathrm{Z}=76$ : no Os II lines were observed.

Iridium (Ir) $\mathrm{Z}=77$ : except for the weak line observed at $2245.750 \AA$, no other Ir II lines can be conclusively identified in the spectrum. From this line we derived an abundance of -11.15 dex, i.e an underabundance of $[-0.5]$. The $\log g f$ values for Ir II were taken from the Kurucz database. None of the Ir II lines listed in Ivarsson et al. (2004) was observed in the spectrum, even assuming a solar abundance. The Ir II line at $3042.553 \AA$ is a minor contributor of a blend with Ti II. The blending was not considered when we analyzed the UVES spectrum, so that we derived a solar iridium abundance (Castelli \& Hubrig 2004).

Platinum (Pt) Z = 78: for Pt II and Pt III we adopted the lines and the transition probabilities listed by Wyart et al. (1995) and by Ryabtsev et al. (1993), respectively. We did not find any clear evidence for a Pt overabundance. Only the Pt II line at $1777.086 \AA$ is predicted with solar abundance and observed. It is a blend with Fe II 1777.058 $\AA$, which is computed as weaker than observed, but an overabundance of platinum gives rise to computed lines not observed in the spectrum. We believe that the line at 4514.124 A, identified as Pt II in the UVES spectrum (Castelli \& Hubrig 2004), is actually due to some other element.

Gold $(\mathrm{Au}) \mathrm{Z}=79$ : gold is overabundant by 3.6 dex. The abundance from the optical region adequately reproduces the numerous Au II lines observed in the ultraviolet. A few of them are unblended, good examples being the Au II lines at 1469.142, $1673.587,1740.475,1793.297$ (blend), and 1800.579 A. There are several predicted Au III lines, but they are all blended, except for those at 1365.382 and $1385.768 \AA$. We adopted the Au II and Au III line lists from Rosberg \& Wyart (1997) and Wyart et al. (1996), respectively.

Mercury $(\mathrm{Hg}) \mathrm{Z}=80$ : the $\log g f$ values for $\mathrm{Hg} \mathrm{I}$ and $\mathrm{Hg}$ II were taken from the NIST database. For $\mathrm{Hg}$ III we considered only the three lines at $1360.509,1647.482$, and $1738.540 \AA$ listed by Profitt et al. (1999). The abundance of -6.60 dex is well suited to reproducing the lines in the ultraviolet, while -6.30 dex derived from the $\mathrm{Hg}$ II line at $3984 \AA$ (Castelli \& Hubrig 2004) is too large. We did not consider any hyperfine structure in the computation of the ultraviolet lines. For $\mathrm{Hg} \mathrm{I}$, the line at $1849.499 \AA$ is blended, while the line at $2536.521 \AA$ is very weak. No other lines with available log $g f$ values were observed. Numerous lines of $\mathrm{Hg}$ II were observed and computed. For instance, the line at $1942.273 \AA$ is strong, but it is blended with Mn II at $1942.344 \AA$. Two lines at $1321.712 \AA$ and $1331.738 \AA$ are single and well reproduced, those at 1354.289 , 1539.142, and $1869.226 \AA$ are strong, but blended. For Hg III, the line at $1360.509 \AA$ is computed as too strong, that at $1647.471 \AA$ would be adequately reproduced if the Mn III line at $1647.497 \AA$ were omitted; finally, the line at $1738.540 \AA$ is blended with numerous other components.

\section{References}

Adelman, S. J. 1994, MNRAS, 266, 97

Ansbacher, W., Pinnington, E. H., Bahr, J. L., \& Kernahan, J. A. 1985, Can. J. Phys., 63, 1330

Asplund, M., Grevesse, N., Sauval, A. J., \& Scott, P. 2009, ARA\&A, 47, 481

Ayres, T. R. 2010, ApJS, 187, 149

Ayres, T. R. 2014, http://casa.colorado.edu/ ayres/ASTRAL/

Bäckström, E., Nilsson, H., Engström, L., Hartman, H., \& Mannervik, S. 2013, J. Phys. B: Atom. Mol. Phys., 46, 205001

Bidelman, W. P. 1962, AJ, 67, 111

Biémont, E., Garnir, H. P., Li, Z. S., et al. 2001, J. Phys. B: Atom. Mol. Phys., 34, 1869

Biémont, É., Blagoev, K., Engström, L., et al. 2011, MNRAS, 414, 3350

Carpenter, K. G., \& Ayres, T. R. 2015, Proc. Conf., Lowell Observatory, 8-14 June, 2014, eds. G. van Belle, \& H. C. Harris, 1041

Castelli, F., \& Bonifacio, P. 1990, A\&AS, 84, 259

Castelli, F., \& Hubrig, S. 2004, A\&A, 425, 263

Castelli, F., \& Kurucz, R. L. 2010, A\&A, 520, A57

Castelli, F., \& Parthasarathy, M. 1995, Astrophysical Applications of Powerful New Databases, ASP Conf. Ser. 78, 151

Castelli, F., Cornachin, M., Morossi, C., \& Hack, M. 1985, A\&AS, 59, 1

Castelli, F., Johansson, S., \& Hubrig, S. 2008, J. Phys. Conf. Ser., 130, 012003

Castelli, F., Kurucz, R. L., \& Hubrig, S. 2009, A\&A, 508, 401

Corliss, C. H., \& Bozman, W. R. 1962, NBS Monograph (Washington: US Department of Commerce, National Bureau of Standards)

Cowan, R. D. 1981, The Theory of Atomic Structure and Spectra (Berkeley: Univ. California Press)

Cowley, C. R., \& Hensberge, H. 1981, ApJ, 244, 252

Den Hartog, E. A., Lawler, J. E., Sobeck, J. S., Sneden, C., \& Cowan, J. J. 2011, ApJS, 194, 35

Dworetsky, M. M. 1969, ApJ, 156, L101

Holt, R. A., Scholl, T. J., \& Rosner, S. D. 1999, MNRAS, 306, 107

Iglesias, L., \& Velasco, R. 1964, The spectrum of the Mn+ ion, eds. L. Iglesias, \& R. Velasco (Madrid: Consejo Superior de Investigaciones Cientificas)

Ivarsson, S., Wahlgren, G. M., Dai, Z., Lundberg, H., \& Leckrone, D. S. 2004, A\&A, 425, 353

Kling, R., \& Griesmann, U. 2000, ApJ, 531, 1173

Kling, R., Schnabel, R., \& Griesmann, U. 2001, ApJS, 134, 173

Kramida, A., \& Sansonetti, J. E. 2013, ApJS, 205, 14

Kramida, A., Ralchenko, Y., Reader, J., \& NIST ASD Team 2014, NIST Atomic Spectra Database, ver. 5.2, http://physics.nist.gov/asd

Kurucz, R. L. 1988, Trans. IAU, XXB, ed. McNally (Dordrecht: Kluwer), 168

Kurucz, R. 1993, SYNTHE Spectrum Synthesis Programs and Line Data. Cdrom No. 18 (Cambridge, Mass.: Smithsonian Astrophysical Observatory), http://kurucz .harvard. edu/linelists/gfall/

Kurucz, R. L. 2005, Mem. Soc. Astron. It. Suppl., 8, 14

Kurucz, R. L. 2011, Canadian J. Phys., 89, 417

Kurucz, R. L. 2014, File hyper250155.pos, available at http://kurucz. harvard.edu/atoms/2501/hyper250155.pos

Lundberg, H., Johansson, S. G., Larsson, J., et al. 1996, ApJ, 469, 388

Moore, C. E. 1950, NBS Circular 488 (Washington: US Government Printing Office)

Nave, G., \& Johansson, S. 2013, ApJS, 204, 1

Nielsen, K. E., Wahlgren, G. M., Proffitt, C. R., Leckrone, D. S., \& Adelman, S. J. 2005, AJ, 130, 2312

Peterson, R. C., \& Kurucz, R. L. 2015, ApJS, 216, 1

Proffitt, C. R., Brage, T., Leckrone, D. S., et al. 1999, ApJ, 512, 942

Quinet, P. 1996, Phys. Scr., 54, 483

Quinet, P., Biémont, E., Palmeri, P., et al. 2012, A\&A, 537, A74

Rosberg, M., \& Wyart, J.-F. 1997, Phys. Scr., 55, 690

Ryabchikova, T. A., Piskunov, N. E., Kupka, F., \& Weiss, W. W. 1997, Balt. Astron., 6, 244, http://vald.inasan.ru/ vald3/php/vald.php

Ryabtsev, A. N., Wyart, J.-F., Joshi, Y. N., Raassen, A. J. J., \& Uylings, P. H. M. 1993, Phys. Scr., 47, 45

Sugar, J., \& Corliss, C. 1985 (Washington: American Chemical Society)

Uylings, P. H. M., \& Raassen, A. J. J. 1997, A\&AS, 125, 539

Warner, B., \& Kirkpatrick, R. C. 1969, MNRAS, 142, 265

Wyart, J.-F., Blaise, J., \& Joshi, Y. N. 1995, Phys. Scr, 52, 535

Wyart, J.-F., Joshi, Y. N., Tchang-Brillet, L., \& Raassen, A. J. J. 1996, Phys. Scr., 53,174 